\title{
DeepSNP: An End-to-End Deep Neural Network with Attention-Based Localization for Breakpoint Detection in Single-Nucleotide Polymorphism Array Genomic Data
}

\author{
HAMID EGHBAL-ZADEH,${ }^{1}$ LUKAS FISCHER ${ }^{2}$ NIKO POPITSCH,${ }^{3}$ FLORIAN KROMP, ${ }^{3}$ \\ SABINE TASCHNER-MANDL, ${ }^{3}$ TERESA GERBER, ${ }^{3}$ EVA BOZSAKY,${ }^{3}$ PETER F. AMBROS, ${ }^{3}$ \\ INGE M. AMBROS, ${ }^{3}$ GERHARD WIDMER, ${ }^{1}$ and BERNHARD A. MOSER ${ }^{2}$
}

\begin{abstract}
Clinical decision-making in cancer and other diseases relies on timely and cost-effective genome-wide testing. Classical bioinformatic algorithms, such as Rawcopy, can support genomic analysis by calling genomic breakpoints and copy-number variations (CNVs), but often require manual data curation, which is error prone, time-consuming, and thus substantially increasing costs of genomic testing and hampering timely delivery of test results to the treating physician. We aimed to investigate whether deep learning algorithms can be used to learn from genome-wide single-nucleotide polymorphism array (SNPa) data and improve state-of-the-art algorithms. We developed, applied, and validated a novel deep neural network (DNN), DeepSNP. A manually curated data set of $50 \mathrm{SNPa}$ analyses was used as truthset. We show that DeepSNP can learn from SNPa data and classify the presence or absence of genomic breakpoints within large genomic windows with high precision and recall. DeepSNP was compared with well-known neural network models as well as with Rawcopy. Moreover, the use of a localization unit indicates the ability to pinpoint genomic breakpoints despite their exact location not being provided while training. DeepSNP results demonstrate the potential of DNN architectures to learn from genomic SNPa data and encourage further adaptation for CNV detection in SNPa and other genomic data types.
\end{abstract}

Keywords: breakpoint detection, deep neural networks, SNPa, weak label.

\section{INTRODUCTION}

C OPY-NUMBER ALTERATIONS (CNAs) such as losses, gains, or amplifications of DNA sequences result in changes in the copy-number status (CNS) of the respective genomic regions. Segmental CNAs, occurring within chromosomes, can range in size from a few base pairs to whole chromosome arms. They are, for example, used to molecularly diagnose and/or stratify cancer patients into risk groups, a prerequisite to

\footnotetext{
${ }^{1}$ Institute of Computational Perception, Johannes Kepler University, Linz, Austria.

${ }^{2}$ Software Comptence Center Hagenberg (SCCH), Hagenberg, Austria.

${ }^{3}$ Children's Cancer Research Insitute (CCRI), Vienna, Austria.
} 
allocate patients to appropriate treatment protocols. Accurate identification of CNAs is thus critical for studying the pathogenesis of cancer and many other diseases.

Today, despite emerging new technologies such as whole-genome sequencing (WGS), microarrays and in particular single-nucleotide polymorphism array (SNPa) are a common choice for copy-number analyses in clinical routine. Reasons for this include their simplicity, robustness, and good cost/benefit ratio. SNPa interrogates a patient genome at defined genomic positions using copy-number and allele-specific oligonucleotide probes.

A core step of copy-number analyses is the segmentation of patient genomes into genomic regions of equal copy-number. Such genomic regions, further called segments, are defined by their endpoints (aka breakpoints), that is, transitions in the CNS of two adjacent segments, and can be determined by considering neighboring probes of an SNPa. These probes provide information about the relative abundance of DNA at a particular genomic position (i.e., its copy-number) and about the directly related ratio of two different alleles at polymorphic positions (the B allele frequency [BAF]) (LaFramboise, 2009).

Accurate copy-number calling from these data requires breakpoint detection, but is challenging for multiple reasons, including mosaicism, technical noise, repetitiveness of the genome, and technology intrinsic biases such as guanine/cytosine (GC) bias [DNA fragments that are very rich/poor in G and C bases show different hybridization/amplification characteristics, cf. Benjamini and Speed (2012)] or probe cross-hybridization.

In this article, we propose a novel end-to-end deep neural network (DNN), namely DeepSNP, specialized to process SNPa data, preprocessed by a tool called Rawcopy, and to predict breakpoints therein. DeepSNP is capable of processing very long stretches of copy-number data by incorporating convolutional layers and benefiting from state-of-the-art feature learning architectures. In addition, it can learn long genomic distance relationships using recurrent layers. Finally, by integrating attention mechanism and localization layers, DeepSNP is capable of accurately pinpointing breakpoints without the need of using accurate labels for training. Breakpoint calling precision is increased within a given genomic window of probes compared with the general purpose state-of-the-art DNNs. Although direct comparison is difficult, DeepSNP also compares well with biological tools such as Rawcopy (Mayrhofer et al., 2016) in predicting the presence of breakpoints in this setting.

\section{RELATED WORK}

Over time, many different algorithms for breakpoint detection have been proposed, mostly relying on statistical methods, including hidden Markov models (HMM) (Marioni et al., 2006; Wang et al., 2007), Bayesian approaches (Pique-Regi et al., 2008; Zhang and Gerstein, 2010), or circular binary segmentation (CBS) (Olshen et al., 2004), a simple recursive method that was shown to outperform other approaches in terms of sensitivity and false discovery rate (Zhao et al., 2013). Briefly, CBS starts by considering a whole chromosome and recursively segments it by applying a simple statistical test (maximal t-statistic) for change-point detection to these segments. CBS stops when no more change points can be found in the current segmentation (Venkatraman and Olshen, 2007). HMM- and CBS-based solutions are implemented in many popular copy-number variation (CNV) tools such as ChAS, Nexus, or Rawcopy and provide relatively accurate and stable copy-number calls.

Current algorithms perform well in routine analysis, when DNA quality and quantity are sufficient, tumor cell content is high, and the respective SNPa data show relatively "clean" profiles. In practice, however, SNPa profiles are often noisy. Reasons for this include crosslinked and/or fragmented DNA, due to tissue preparation procedures such as formalin fixation, or unusual long storage of tissue/DNA. Furthermore, when DNA amounts are limited, such as in cell-free DNA extractions from liquid biopsies, low DNA input and contamination with nontumor DNA can also contribute to the noise in the data. With current algorithms, noisy and/or subclonal/contaminated samples often result in reduced segmentation accuracy, mainly due to increased false positive rates, and/or high fragmentation (i.e., many small neighboring segments of alternating copy-number). Such segments require manual curation, which is work intensive and error prone. Thus, there is a need to develop algorithms predicting breakpoints and genomic segments with higher precision from these data.

Deep learning (DL) methods outperformed classical state-of-the-art algorithms across various domains, including image classification, speech recognition, language translation, or document analysis, and were 
recently also successfully applied to various problems in the life science domain (Angermueller et al., 2016). DL architectures can learn unknown or hidden relationships from highly complex and noisy data if a sufficient amount of training data are available, even if only weak labels can be provided (Ching, 2018). DeepVariant, a DL architecture to predict SNPs or small insertions/deletions (indels) in WGS data, was proposed by Poplin et al. (2016). Although results show that DeepVariant can learn the statistical relationship between aligned reads and true SNPs/indels over various sequencing technologies, the architecture is not directly applicable to call CNVs from SNPa data as technology and thus data structure and resolution differ substantially. Moreover, the architecture is a pure classification approach relying on currently available frameworks calling candidate predictions.

Thus, DeepVariant is not suitable to further call CNVs. Most recently, Gupta and Rush (2017) proposed to use dilated convolution on regulatory marker locations from ENCODE (Consortium, 2012) to model long-distance genomic dependencies. They showed that dilated convolution can outperform long shortterm memory (LSTM) (Hochreiter and Schmidhuber, 1997) recurrent models. Nevertheless, they did not evaluate their method on the detection of rare genomic events such as breakpoints. Furthermore, their method requires long input sequences and does not have a localization ability.

\section{DATA COLLECTION AND PREPROCESSING}

We analyzed 50 samples of 18 neuroblastoma patients (tissue source was tumor, bone marrow disseminated tumor cells, bone marrow nontumor cells, or cell line) on the Affymetrix CytoScan HD SNP array platform (Ambros et al., 2014). The resulting CEL files contain $~ 2.8$ Mio raw array intensity values converted into normalized $\log \mathrm{R}$ ratio (LRR) and BAF values using Rawcopy, an open $\mathrm{R}$ package for processing Affymetrix microarray data (Mayrhofer et al., 2016) (Fig. 1b). Both measures are then further normalized using observed per-probe value distributions derived from a large set of reference samples.

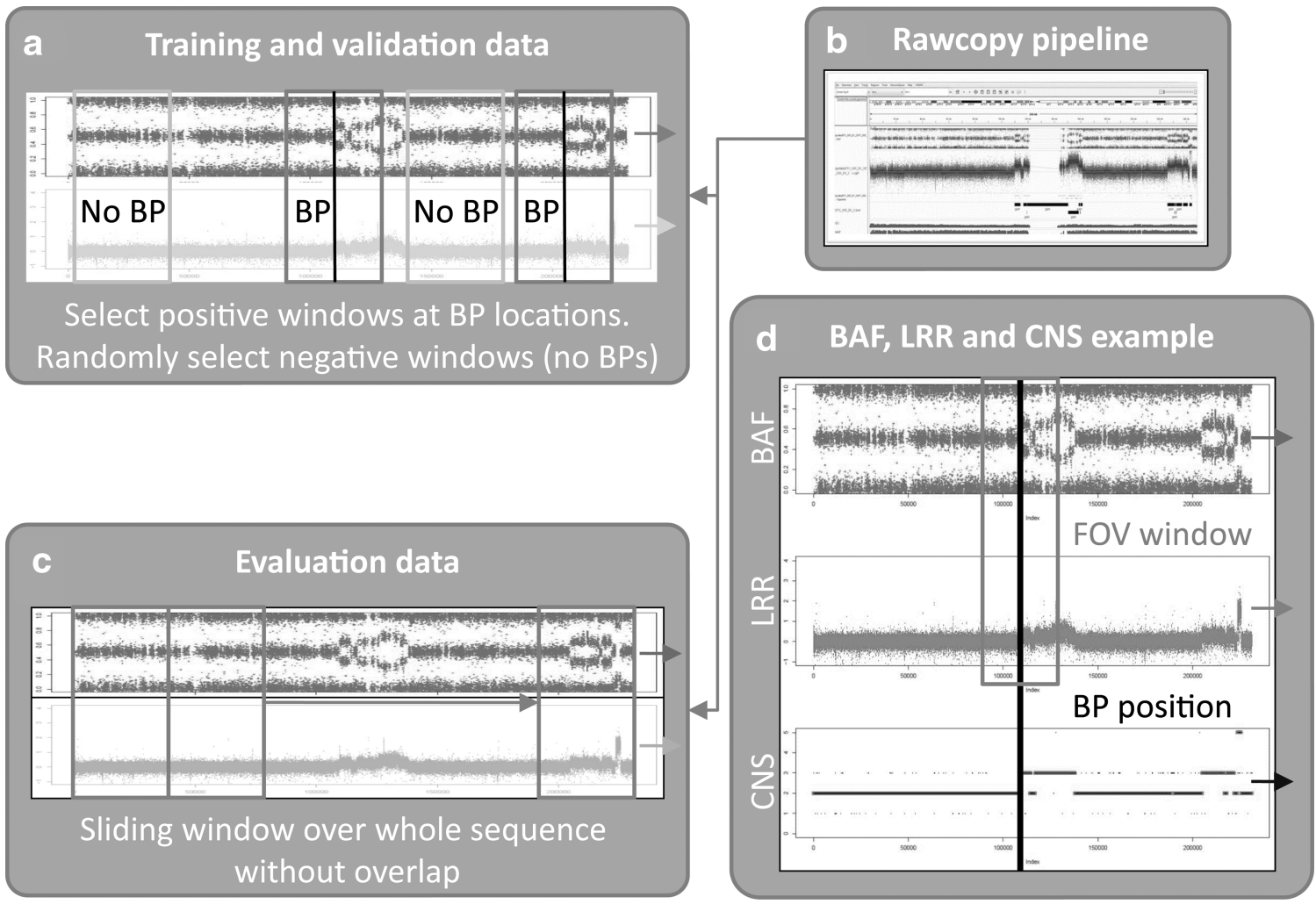

FIG. 1. Data generation pipeline. All images show part of a sample sequence with $\sim 225,000$ values. (a) Training data generation. (b) Example UI (IGV/VARAN-GIE) of the Rawcopy pipeline. (c) Evaluation data generation. (d) Example window (grey rectangle) placed at a breakpoint position (vertical black line). CNS in the last row depicts the manual truthset CNS (grey horizontal lines) as well as the Rawcopy CNS (black horizontal lines and dots). CNS, copy-number status. 
LRR values are corrected for fragment length and GC content. Finally, Rawcopy calls breakpoints using an allele-specific CBS algorithm and outputs text files containing normalized LRR and BAF values as well as called copy-number segments. These resulting data files contained 2,819,443 LRR values and around 480, 000 BAF values each (Fig. 1a), which were then considered input data for our method.

To create the ground truth, we manually curated the Rawcopy predictions using our in-house editor Varan-GIE (Popitsch, 2018), taking additional data files (such as related data sets, mappability tracks, and annotations of common CNVs) into account. Highly fragmented segments often occur in noisy profiles as artifacts predicted by Rawcopy and need to be merged. In case of false negative predictions, additional segments have to be generated and added to the final segmentation. More frequently, due to a high sensitivity but lower specificity of Rawcopy predictions, false positive segments have to be removed. The manually curated segments were called by experienced biologists and always doublechecked by at least one additional person (according to laboratory routine) and considered the truth-set in the following (Fig. 1d).

To train our network, we combined the Rawcopy output, our manual curation set, and additional genome-wide data to create genome-wide data files that contain the following data. (1) Genomic position (chromosome+offset) of the respective SNPa probe, (2) Rawcopy LRR, (3) Rawcopy BAF (-1 encoding missing values), (4) Encoded truth-set CNS (normal, loss, gain, amplification) at the probe position, and (5) Encoded Rawcopy CNS at the probe position (used in our evaluation).

\subsection{Data preparation for deep learning}

The samples of SNPa are very long ( $\sim 2.8$ Mio values) and cannot be directly fed to a neural network. Therefore, we designed a windowing scheme to create training and validation data. Based on the truth-set of CNS, we select positive and negative windows of probes and use them as training and validation data. A window of $n$ probes is considered positive, if the CNS changes at least once or negative, if the CNS does not change at all. The change of CNS (CNS transition) represents a breakpoint; therefore, a positive window is defined as a window with at least one breakpoint.

For each genomic sample, we select the positive windows as follows. First, we locate the position of all breakpoints in that sample based on the truth-set. Second, a window of $n$ probes is centered at all the known breakpoint positions one by one. Due to the possible small distance between neighboring breakpoints, positive windows can contain multiple breakpoints. To select negative windows, we randomly select (nonoverlapping) windows with $n$ consecutive probes that do not contain any breakpoints.

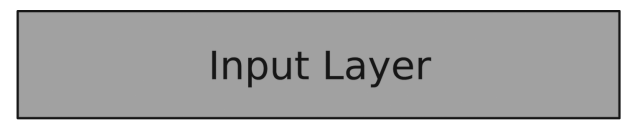

Feature Learning Unit

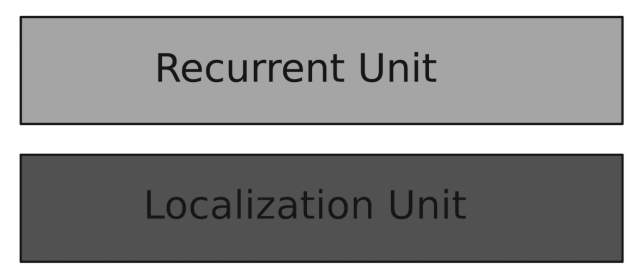

Attention Unit

Averaging Layer
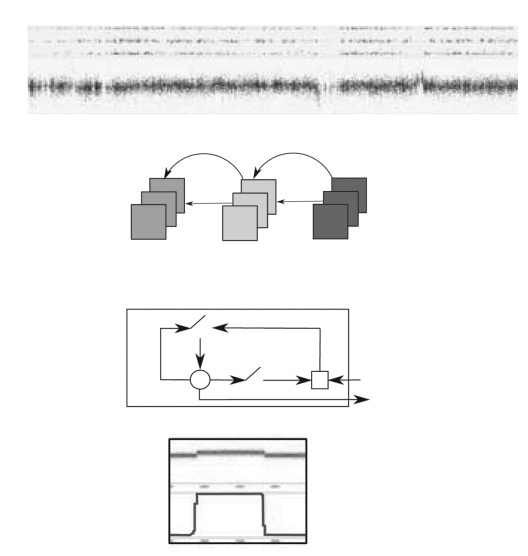

o o o o o 0

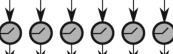

ó ó ó ó ó

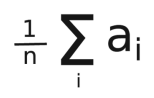

FIG. 2. Block diagram of the proposed DeepSNP architecture. 


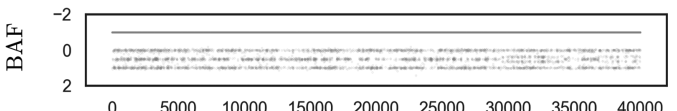

3
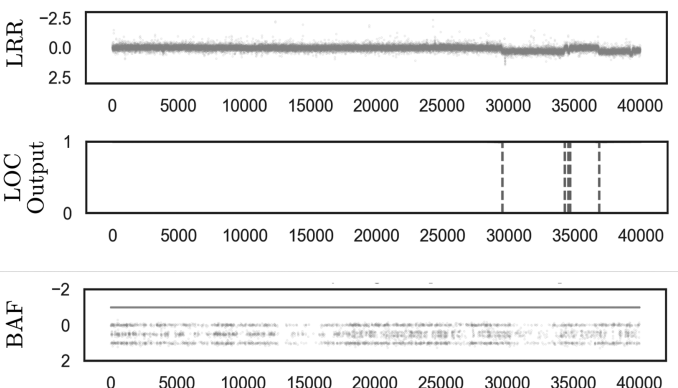

尔
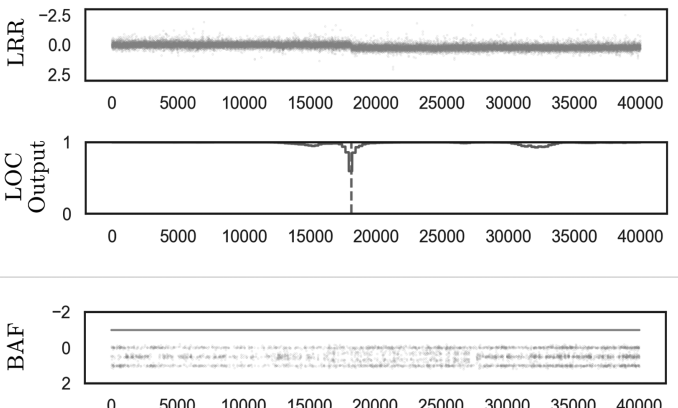

胥

$\begin{array}{llllllll}0 & 5000 & 10000 & 15000 & 20000 & 25000 & 30000 & 35000 \quad 40000\end{array}$
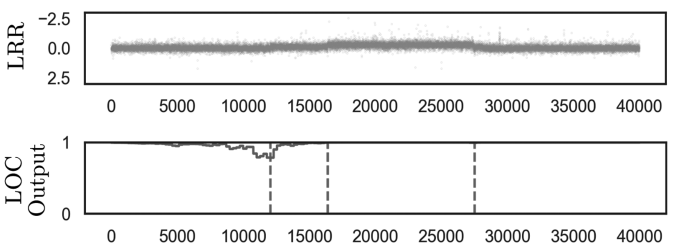

No Attention
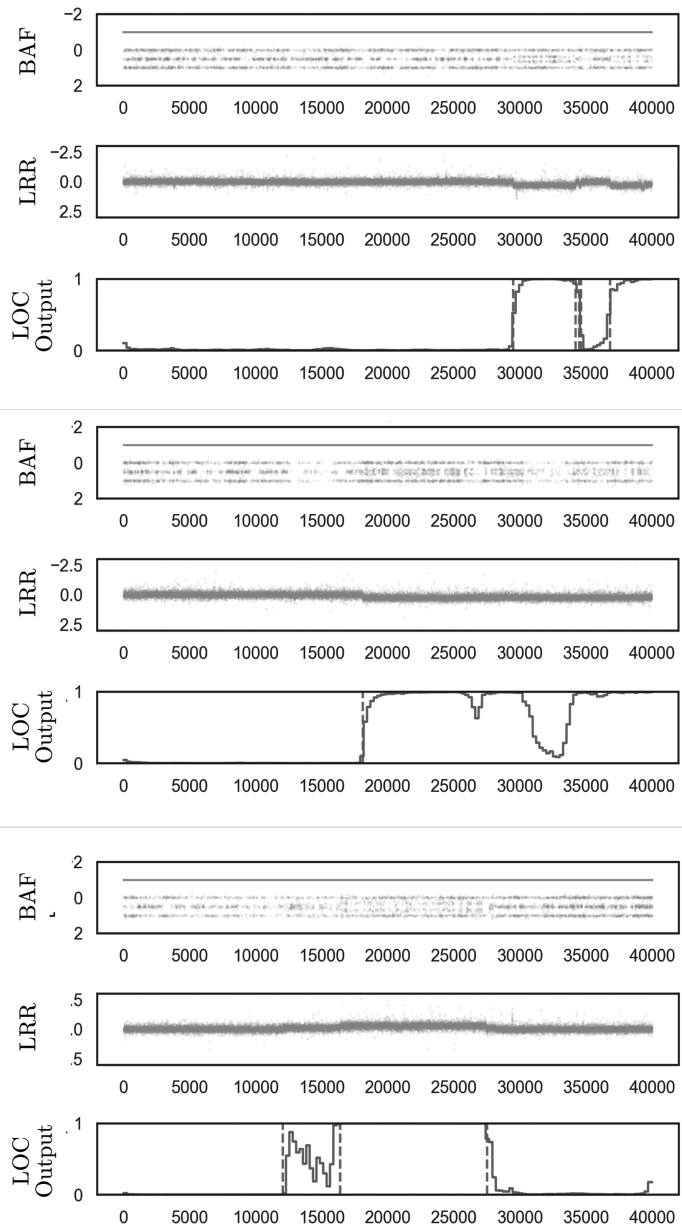

Final Attention

FIG. 3. Example predictions of the localization unit with No (left) and Final (right) Attention from DeepSNPV1. The models were trained only with whether or not a breakpoint exists in a 40k window. The first row shows the BAF values, the second the LRR values, and the third the actual Localization Unit output for a given window. The dashed vertical lines represent the truth-set for breakpoints. All examples were classified correctly. LRR, normalized log R ratio.

LRR and BAF values were concatenated in each window and added to create a $2 \times n$ feature vector. No further information (except breakpoint coordinates extracted at positions of CNS transition) such as chromosome position was taken into account for window selection. Finally, each feature vector was labeled as "has breakpoint(s)/positive," if at least one breakpoint is located within the window or "has no breakpoint(s)/negative," otherwise. The training and validation data selection is depicted in Figure 1a. The evaluation data set is created by sliding a window with no overlap over the whole sample sequence as shown in Figure 1c (for better presentability, only a small subset is shown).

For training, validation, and evaluation, we chose four different window sizes of $n=[40 k, 20 k, 10 k, 5 k]$ probes (Section 5.1).

TABLe 1. InFormation FOR Evaluating THE LOCALIZATION OUTPUTS

\begin{tabular}{ll}
\hline Train WinSize & Evaluation WinSize \\
\hline $40 \mathrm{k}$ & $20 \mathrm{k}, 10 \mathrm{k}, 5 \mathrm{k}$ \\
$20 \mathrm{k}$ & $10 \mathrm{k}, 5 \mathrm{k}$ \\
$10 \mathrm{k}$ & $5 \mathrm{k}$ \\
\hline
\end{tabular}


Table 2. Cross-Validation Results for Baseline Experiments with Window Size 40K

\begin{tabular}{|c|c|c|c|c|c|c|}
\hline \multicolumn{7}{|c|}{ Window size: $40 k$} \\
\hline$(\%) / F o l d s$ & Fold 1 & Fold 2 & Fold 3 & Fold 4 & Fold 5 & Fold 6 \\
\hline \multicolumn{7}{|l|}{ Rawcopy } \\
\hline F1 MAC & 50.69 & 40.55 & 40.35 & 47.72 & 38.66 & 35.22 \\
\hline F1 POS & 22.22 & 36.36 & 49.59 & 50.67 & 45.60 & 61.07 \\
\hline PREC MAC & 55.39 & 60.21 & 64.85 & 66.96 & 64.75 & 71.98 \\
\hline PREC POS & 12.99 & 22.36 & 33.15 & 33.93 & 29.51 & 43.96 \\
\hline REC MAC & 71.71 & 63.14 & 58.47 & 64.42 & 59.44 & 52.45 \\
\hline REC POS & 76.92 & 97.30 & 98.39 & 100.0 & 100.0 & 100.0 \\
\hline \multicolumn{7}{|l|}{ BLVGG } \\
\hline F1 MAC & 54.38 & 56.63 & 50.78 & 47.82 & 42.28 & 36.42 \\
\hline F1 POS & 18.18 & 37.29 & 46.39 & 16.47 & 0.0 & 0.0 \\
\hline PREC MAC & 53.98 & 57.90 & 56.55 & 48.99 & 37.14 & 28.64 \\
\hline PREC POS & 12.90 & 27.16 & 34.09 & 25.0 & 0.0 & 0.0 \\
\hline REC MAC & 58.63 & 62.97 & 57.48 & 49.41 & 49.06 & 50.0 \\
\hline REC POS & 30.77 & 59.46 & 72.58 & 12.28 & 0.0 & 0.0 \\
\hline \multicolumn{7}{|l|}{ BLDenseNet } \\
\hline F1 MAC & 54.05 & 63.95 & 50.14 & 49.03 & 59.15 & 41.69 \\
\hline F1 POS & 11.76 & 37.04 & 16.90 & 12.90 & 30.77 & 10.20 \\
\hline PREC MAC & 59.63 & 72.52 & 69.61 & 77.26 & 84.56 & 64.84 \\
\hline PREC POS & 25.0 & 58.82 & 66.67 & 80.0 & 90.91 & 71.43 \\
\hline REC MAC & 53.10 & 61.52 & 53.85 & 53.19 & 58.94 & 51.93 \\
\hline REC POS & 7.69 & 27.03 & 9.68 & 7.02 & 18.52 & 5.49 \\
\hline \multicolumn{7}{|c|}{ BLDilated-DenseNet } \\
\hline F1 MAC & 79.32 & 59.19 & 85.82 & 89.39 & 85.09 & 83.48 \\
\hline F1 POS & 60.87 & 45.39 & 78.43 & 84.11 & 76.40 & 80.46 \\
\hline PREC MAC & 83.52 & 63.09 & 93.64 & 91.32 & 92.95 & 84.09 \\
\hline PREC POS & 70.0 & 30.77 & 100.0 & 90.0 & 97.14 & 84.34 \\
\hline REC MAC & 76.17 & 72.79 & 82.26 & 87.87 & 81.17 & 83.13 \\
\hline REC POS & 53.85 & 86.49 & 64.52 & 78.95 & 62.96 & 76.92 \\
\hline \multicolumn{7}{|c|}{ BLLSTM-DenseNet } \\
\hline F1 MAC & 76.04 & 77.14 & 83.01 & 89.82 & 81.38 & 84.99 \\
\hline F1 POS & 54.55 & 64.58 & 74.0 & 84.62 & 70.45 & 81.25 \\
\hline PREC MAC & 81.62 & 74.32 & 91.54 & 92.89 & 89.16 & 88.07 \\
\hline PREC POS & 66.67 & 52.54 & 97.37 & 93.62 & 91.18 & 94.20 \\
\hline REC MAC & 72.33 & 83.94 & 79.51 & 87.63 & 77.76 & 84.07 \\
\hline REC POS & 46.15 & 83.78 & 59.68 & 77.19 & 57.41 & 71.43 \\
\hline
\end{tabular}

All values are shown in \%.

3.1.1. Weakly labeled annotations. Since we do not provide the exact position of the breakpoint within a window of probes during training, our data are weakly labeled (Sun et al., 2010). Conventional DNNs are not capable of predicting with higher resolution than their input, and therefore, they can only predict one label for a fixed window of given inputs. In contrast, DeepSNP is capable of predicting the labels with higher resolutions than its input data window due to its Localization Unit. Hence, it can predict breakpoints within a window of probes it receives as an input with very fine resolution.

\section{DEEPSNP: AN END-TO-END NEURAL NETWORK FOR BREAKPOINT DETECTION IN SINGLE-NUCLEOTIDE POLYMORPHISM ARRAY}

The genomic data considered by our approach are characterized by very long sequences of lowdimensional data, making analysis with conventional DL architectures difficult. In our empirical results, we show that state-of-the-art architectures such as $\mathrm{VGG}^{1}$ (Simonyan and Zisserman, 2014) and DenseNet

\footnotetext{
${ }^{1}$ An architecture proposed by the Visual Geometry Group, University of Oxford.
} 
Table 3. Cross-Validation Results for DeepSNP Experiments with Window Size 40K

\begin{tabular}{lcccccc}
\hline \multicolumn{7}{c}{ Window size: $40 k$} \\
\hline (\%)/Folds & Fold 1 & Fold 2 & Fold 3 & Fold 4 & Fold 5 & Fold 6 \\
\hline DeepSNPv1 (no attention) & & & & & \\
F1 MAC & 91.07 & 89.07 & 71.35 & 88.50 & 88.45 & 82.24 \\
F1 POS & 85.95 & 81.72 & 54.26 & 82.03 & 85.24 & 72.73 \\
PREC MAC & 93.42 & 90.89 & 68.64 & 87.43 & 87.60 & 81.20 \\
PREC POS & 92.44 & 86.36 & 40.70 & 78.76 & 80.10 & 69.23 \\
REC MAC & 89.19 & 87.50 & 81.48 & 89.74 & 89.76 & 83.48 \\
REC POS & 80.31 & 77.55 & 81.40 & 85.58 & 91.07 & 76.60 \\
DeepSNPv1 (final attention) & & & & & \\
F1 MAC & 92.53 & 86.42 & 73.30 & 89.32 & 86.22 & 78.68 \\
F1 POS & 88.25 & 77.93 & 57.25 & 83.09 & 82.70 & 68.97 \\
PREC MAC & 94.87 & 84.43 & 70.21 & 89.46 & 85.33 & 76.55 \\
PREC POS & 94.69 & 72.17 & 43.20 & 83.50 & 75.74 & 57.97 \\
REC MAC & 90.63 & 88.94 & 83.76 & 89.18 & 88.09 & 83.82 \\
REC POS & 82.63 & 84.69 & 84.88 & 82.69 & 91.07 & 85.11 \\
DeepSNPv2 (no attention) & & & & & & \\
F1 MAC & 88.55 & 82.33 & 78.48 & 86.94 & 93.13 & 84.61 \\
F1 POS & 81.74 & 69.33 & 62.86 & 78.69 & 90.74 & 75.0 \\
PREC MAC & 92.97 & 95.54 & 78.08 & 91.74 & 94.04 & 90.73 \\
PREC Pos & 93.53 & 100.0 & 61.80 & 91.14 & 94.23 & 90.91 \\
REC MAC & 85.55 & 76.53 & 78.90 & 83.72 & 92.38 & 81.01 \\
REC Pos & 72.59 & 53.06 & 63.95 & 69.23 & 87.50 & 63.83 \\
DeepSNPv2 (final attention) & & & & & \\
F1 MAC & 88.67 & 84.85 & 78.71 & 87.81 & 94.43 & 86.41 \\
F1 POS & 81.92 & 73.89 & 63.22 & 80.21 & 92.41 & 78.05 \\
PREC MAC & 93.21 & 95.22 & 78.44 & 91.68 & 96.17 & 91.50 \\
PREC POS & 94.0 & 98.31 & 62.50 & 90.36 & 98.65 & 91.43 \\
REC MAC & 85.61 & 79.49 & 78.99 & 85.04 & 93.15 & 83.14 \\
REC POS & 72.59 & 59.18 & 63.95 & 72.12 & 86.90 & 68.09 \\
\hline
\end{tabular}

All values are shown in \%.

(Huang et al., 2016) that perform very well in audio (Eghbal-zadeh et al., 2016; Hershey et al., 2017) and image processing (Simonyan and Zisserman, 2014; Huang et al., 2016) applications are not capable of coping well with these data. In addition, we adapt the DenseNet architectures to the task at hand to improve their performance and hence have more competitive baselines. Based on the DenseNet architecture, we introduce LSTM-DenseNet and Dilated-DenseNet that learn very well from SNPa data and outperform their conventional counterparts.

Besides improving the available baselines, we introduce DeepSNP-a novel DNN with specialized units, targeting specific processing goals-to deal with the challenges of breakpoint detection in SNPa. DeepSNP is capable of detecting the presence or absence of a breakpoint within a window of probes and outperforms all the baselines. Using its Localization Unit, DeepSNP can generate breakpoint annotations for the probes within each window without having access to the exact position of breakpoints within the window during training. In the following section, we detail the architecture and different DeepSNP units.

\subsection{Network architecture}

DeepSNP (Fig. 2) is a DNN trained with stochastic gradient descent (SGD) in an end-to-end manner. It has four main units, each playing an important role for learning a suitable hidden representation from the genomic data and further learning the relevant aspects of a breakpoint from the long, low-dimensional input sequence. The processing units of DeepSNP are detailed as follows. 
Table 4. Cross-Validation Results for Baseline Experiments with Window Size 20K

\begin{tabular}{|c|c|c|c|c|c|c|}
\hline \multicolumn{7}{|c|}{ Window size: $20 k$} \\
\hline$(\%) /$ Folds & Fold 1 & Fold 2 & Fold 3 & Fold 4 & Fold 5 & Fold 6 \\
\hline \multicolumn{7}{|l|}{ Rawcopy } \\
\hline F1 MAC & 51.66 & 36.13 & 39.88 & 47.19 & 41.45 & 28.48 \\
\hline F1 POS & 17.60 & 23.15 & 37.75 & 36.95 & 33.42 & 40.49 \\
\hline PREC MAC & 54.47 & 55.80 & 61.13 & 61.33 & 60.03 & 62.69 \\
\hline PREC POS & 9.91 & 13.18 & 23.33 & 22.66 & 20.07 & 25.38 \\
\hline REC MAC & 77.06 & 63.92 & 62.69 & 70.14 & 66.44 & 54.49 \\
\hline REC POS & 78.57 & 95.12 & 98.72 & 100.0 & 100.0 & 100.0 \\
\hline \multicolumn{7}{|l|}{ BLVGG } \\
\hline F1 MAC & 52.77 & 47.32 & 60.91 & 48.39 & 53.48 & 43.30 \\
\hline F1 POS & 13.70 & 0.0 & 38.92 & 5.56 & 23.13 & 0.0 \\
\hline PREC MAC & 53.00 & 45.13 & 60.18 & 53.74 & 53.36 & 38.18 \\
\hline PREC POS & 8.47 & 0.0 & 33.64 & 22.22 & 19.77 & 0.0 \\
\hline REC MAC & 61.26 & 49.74 & 62.79 & 50.62 & 54.40 & 50.0 \\
\hline REC POS & 35.71 & 0.0 & 46.15 & 3.17 & 27.87 & 0.0 \\
\hline \multicolumn{7}{|l|}{ BLDenseNet } \\
\hline F1 MAC & 60.98 & 67.65 & 57.31 & 68.61 & 52.50 & 46.28 \\
\hline F1 POS & 23.53 & 40.58 & 23.91 & 43.37 & 12.31 & 5.77 \\
\hline PREC MAC & 81.90 & 71.58 & 81.10 & 89.42 & 93.20 & 75.92 \\
\hline PREC POS & 66.67 & 50.0 & 78.57 & 90.0 & 100.0 & 75.0 \\
\hline REC MAC & 57.02 & 65.24 & 56.62 & 64.01 & 53.28 & 51.35 \\
\hline REC POS & 14.29 & 34.15 & 14.10 & 28.57 & 6.56 & 3.0 \\
\hline \multicolumn{7}{|c|}{ BLDilated-DenseNet } \\
\hline F1 MAC & 73.21 & 59.09 & 81.60 & 87.89 & 87.11 & 80.11 \\
\hline F1 POS & 48.0 & 36.65 & 69.12 & 78.90 & 78.13 & 70.14 \\
\hline PREC MAC & 76.30 & 60.57 & 86.27 & 94.09 & 85.77 & 79.17 \\
\hline PREC POS & 54.55 & 23.33 & 81.03 & 93.48 & 74.63 & 66.67 \\
\hline REC MAC & 70.82 & 77.63 & 78.53 & 83.71 & 88.64 & 81.27 \\
\hline REC POS & 42.86 & 85.37 & 60.26 & 68.25 & 81.97 & 74.0 \\
\hline \multicolumn{7}{|c|}{ BLLSTM-DenseNet } \\
\hline F1 MAC & 75.42 & 75.13 & 80.93 & 85.98 & 84.82 & 81.97 \\
\hline F1 POS & 52.17 & 56.31 & 67.23 & 75.47 & 73.27 & 70.81 \\
\hline PREC MAC & 82.37 & 71.73 & 93.81 & 93.49 & 93.12 & 90.78 \\
\hline PREC POS & 66.67 & 46.77 & 97.56 & 93.02 & 92.50 & 93.44 \\
\hline REC MAC & 71.06 & 81.05 & 75.50 & 81.33 & 79.91 & 77.88 \\
\hline REC POS & 42.86 & 70.73 & 51.28 & 63.49 & 60.66 & 57.0 \\
\hline
\end{tabular}

All values are shown in \%.

4.1.1. Feature learning unit. In this unit, we incorporate and adapt state-of-the-art convolutional architectures, which can efficiently learn a high-level representation. We decided to choose DenseNet (Huang et al., 2016) due to its performance in areas such as audio and image processing (Takahashi and Mitsufuji, 2017). We modified DenseNet to use only one-dimensional (1D) filters and 1D pooling layers to learn from the very long genomic position dimension.

4.1.2. Distributed recurrent unit. Recurrent models such as LSTM (Hochreiter and Schmidhuber, 1997) and gated recurrent units (GRUs) (Chung et al., 2014) are used to model sequential data such as audio, text, and genome sequences (Gupta and Rush, 2017). Therefore, we use a bidirectional GRU and apply it on the hidden space learned by our Feature Learning Unit. We prefer GRU over LSTM because of its simplicity, fast training, and competitive performance versus LSTM. We apply the GRUs on the sequence of hidden activations formed by the dimension in the hidden space that represents the genomic position of the features. As the filters used are 1D, the dimension in the hidden representation that represents the genomic position can be traced back to the input and represent its corresponding input probes. 
Table 5. Cross-Validation Results for DeepSNP Experiments with Window Size 20K

\begin{tabular}{|c|c|c|c|c|c|c|}
\hline \multicolumn{7}{|c|}{ Window size: $20 k$} \\
\hline$(\%) /$ Folds & Fold 1 & Fold 2 & Fold 3 & Fold 4 & Fold 5 & Fold 6 \\
\hline \multicolumn{7}{|c|}{ DeepSNPv1 (no attention) } \\
\hline F1 MAC & 89.83 & 79.32 & 61.99 & 80.99 & 84.86 & 77.62 \\
\hline F1 POS & 82.48 & 63.11 & 36.71 & 67.77 & 76.43 & 61.90 \\
\hline PREC MAC & 89.00 & 76.75 & 60.93 & 76.87 & 82.11 & 74.28 \\
\hline PREC POS & 80.43 & 56.62 & 23.56 & 55.74 & 67.07 & 52.0 \\
\hline REC MAC & 90.70 & 82.76 & 80.72 & 88.56 & 89.28 & 83.40 \\
\hline REC POS & 84.64 & 71.30 & 82.98 & 86.44 & 88.83 & 76.47 \\
\hline \multicolumn{7}{|c|}{ DeepSNPv1 (final attention) } \\
\hline F1 MAC & 85.70 & 84.97 & 69.38 & 82.56 & 84.24 & 76.36 \\
\hline F1 POS & 75.79 & 72.73 & 46.63 & 70.43 & 75.62 & 60.15 \\
\hline PREC MAC & 82.74 & 86.07 & 65.51 & 78.22 & 81.32 & 72.78 \\
\hline PREC POS & 67.78 & 75.25 & 32.76 & 57.92 & 65.25 & 48.78 \\
\hline REC MAC & 89.77 & 83.96 & 83.79 & 90.48 & 89.31 & 83.57 \\
\hline REC POS & 85.95 & 70.37 & 80.85 & 89.83 & 89.89 & 78.43 \\
\hline \multicolumn{7}{|c|}{ DeepSNPv2 (no attention) } \\
\hline F1 MAC & 83.61 & 76.79 & 73.52 & 85.06 & 86.68 & 78.84 \\
\hline F1 POS & 70.89 & 56.77 & 51.43 & 73.10 & 78.03 & 61.73 \\
\hline PREC MAC & 91.89 & 93.85 & 71.54 & 93.04 & 89.52 & 88.36 \\
\hline PREC POS & 89.95 & 93.62 & 46.55 & 91.14 & 85.44 & 83.33 \\
\hline REC MAC & 78.74 & 70.22 & 76.09 & 80.11 & 84.46 & 73.84 \\
\hline REC POS & 58.50 & 40.74 & 57.45 & 61.02 & 71.81 & 49.02 \\
\hline \multicolumn{7}{|c|}{ DeepSNPv2 (final attention) } \\
\hline F1 MAC & 84.14 & 79.83 & 74.44 & 85.45 & 87.15 & 78.39 \\
\hline F1 POS & 71.83 & 62.58 & 53.62 & 73.89 & 78.86 & 60.98 \\
\hline PREC MAC & 92.67 & 93.71 & 70.97 & 91.73 & 89.56 & 87.01 \\
\hline PREC POS & 91.41 & 92.73 & 44.68 & 88.24 & 85.19 & 80.65 \\
\hline REC MAC & 79.14 & 73.42 & 80.19 & 81.20 & 85.20 & 73.70 \\
\hline REC Pos & 59.15 & 47.22 & 67.02 & 63.56 & 73.40 & 49.02 \\
\hline
\end{tabular}

All values are shown in $\%$.

4.1.3. Localization unit. Localization Units can provide a mechanism to trace a hidden representation back to the input. By using distributed units, we can repeat a processing unit such as softmax on every node of a specific dimension. This way, the predictions of each node in that dimension represent the corresponding indexes in the input (directly mapping to the respective genomic position).

4.1.4. Attention unit. Biologists often have to spend hours investigating the probes carefully to be able to annotate breakpoints as the truth-set. They need to use special tools to explore such long sequences, enabling them to zoom-in and zoom-out in specific regions of the genomic data to make the final decision about a breakpoint. Attention (Vaswani et al., 2017; Xu et al., 2017) is a mechanism that can be used in DNNs to allow the network to attend on specific parts of the activations of a layer. This enables the network to give more/less importance to specific regions of activations in a layer. We use an Attention Unit that allows our network to attend on any activation corresponding to the genomic probes. This enables DeepSNP to explore the probes, focus on specific regions of the genomic sequence if necessary, and process the data in similar ways as the biologist annotators.

We introduce two variants of DeepSNP: (1) with dilated convolution layers (DeepSNPv1) and (2) with conventional convolution layers and no dilation (DeepSNPv2). Dilated convolution layers (Yu and Koltun, 2015) are specialized layers with large receptive fields that can process very long inputs. These layers are known for their significant performance in audio processing (Van Den Oord et al., 2016), machine translation (Kalchbrenner et al., 2016), and for modeling long-distance genomic dependencies (Gupta and Rush, 2017). DeepSNPv1 incorporates dilated convolution in the first three layers to have a large enough 
Table 6. Cross-Validation Results for Baseline Experiments with Window Size 10K

\begin{tabular}{|c|c|c|c|c|c|c|}
\hline \multicolumn{7}{|c|}{ Window size: $10 k$} \\
\hline$(\%) / F o l d s$ & Fold 1 & Fold 2 & Fold 3 & Fold 4 & Fold 5 & Fold 6 \\
\hline \multicolumn{7}{|l|}{ Rawcopy } \\
\hline F1 MAC & 50.82 & 35.98 & 42.99 & 49.31 & 43.94 & 30.72 \\
\hline F1 POS & 12.16 & 15.09 & 27.36 & 28.28 & 24.13 & 27.69 \\
\hline PREC MAC & 53.07 & 53.78 & 57.50 & 58.23 & 56.86 & 58.03 \\
\hline PREC POS & 6.58 & 8.19 & 15.94 & 16.47 & 13.72 & 16.07 \\
\hline REC MAC & 79.91 & 67.69 & 69.09 & 77.13 & 73.40 & 60.15 \\
\hline REC POS & 78.57 & 95.56 & 96.55 & 100.0 & 100.0 & 100.0 \\
\hline \multicolumn{7}{|l|}{ BLVGG } \\
\hline F1 MAC & 49.52 & 45.11 & 57.16 & 50.59 & 18.43 & 46.42 \\
\hline F1 POS & 0.0 & 13.77 & 21.05 & 9.66 & 15.25 & 0.0 \\
\hline PREC MAC & 49.17 & 52.04 & 60.65 & 50.58 & 52.13 & 43.37 \\
\hline PREC POS & 0.0 & 7.86 & 30.43 & 9.33 & 8.30 & 0.0 \\
\hline REC MAC & 49.88 & 59.49 & 55.94 & 50.62 & 53.06 & 49.93 \\
\hline REC POS & 0.0 & 55.56 & 16.09 & 10.0 & 93.94 & 0.0 \\
\hline \multicolumn{7}{|l|}{ BLDenseNet } \\
\hline F1 MAC & 56.28 & 59.16 & 60.91 & 54.66 & 54.54 & 46.25 \\
\hline F1 POS & 13.33 & 26.09 & 26.67 & 13.33 & 13.16 & 0.0 \\
\hline PREC MAC & 99.23 & 57.10 & 84.48 & 96.14 & 71.35 & 43.33 \\
\hline PREC POS & 100.0 & 18.10 & 77.78 & 100.0 & 50.0 & 0.0 \\
\hline REC MAC & 53.57 & 67.40 & 57.78 & 53.57 & 53.47 & 49.59 \\
\hline REC POS & 7.14 & 46.67 & 16.09 & 7.14 & 7.58 & 0.0 \\
\hline \multicolumn{7}{|c|}{ BLDilated-DenseNet } \\
\hline F1 MAC & 62.53 & 51.59 & 83.05 & 74.59 & 83.04 & 75.11 \\
\hline F1 POS & 26.09 & 21.09 & 69.23 & 55.86 & 68.70 & 56.76 \\
\hline PREC MAC & 66.01 & 55.12 & 87.01 & 69.82 & 83.27 & 75.31 \\
\hline PREC POS & 33.33 & 12.31 & 78.26 & 40.79 & 69.23 & 57.27 \\
\hline REC MAC & 60.35 & 72.0 & 80.05 & 88.49 & 82.81 & 74.92 \\
\hline REC POS & 21.43 & 73.33 & 62.07 & 88.57 & 68.18 & 56.25 \\
\hline \multicolumn{7}{|c|}{ BLLSTM-DenseNet } \\
\hline F1 MAC & 83.06 & 60.70 & 84.54 & 90.33 & 84.44 & 82.90 \\
\hline F1 POS & 66.67 & 31.67 & 71.72 & 82.27 & 70.91 & 69.74 \\
\hline PREC MAC & 84.32 & 59.20 & 92.61 & 90.07 & 92.63 & 88.08 \\
\hline PREC POS & 69.23 & 19.89 & 89.66 & 81.69 & 88.64 & 81.93 \\
\hline REC MAC & 81.90 & 80.09 & 79.49 & 90.59 & 79.22 & 79.34 \\
\hline REC POS & 64.29 & 77.78 & 59.77 & 82.86 & 59.09 & 60.71 \\
\hline
\end{tabular}

All values are shown in \%.

receptive field to model dependencies across all input probes. In addition, we provide the results with (Final Attention) and without (No Attention) the Attention Unit to demonstrate its effect on the performance of the predictions.

\section{RESULTS}

In this section, the empirical results for SNPa breakpoint detection using different methods and window sizes are provided. Furthermore, the evaluation of the DeepSNP Localization Unit predictions is given.

\subsection{Evaluation}

All algorithms were evaluated using sixfold cross-validation. In each fold, the samples of 15 patients are used for training and the samples of the three remaining patients are used for validation. For model selection during training, a subset of positive and negative windows was used. All evaluation 
Table 7. Cross-Validation Results for DeepSNP Experiments with Window Size 10K

\begin{tabular}{|c|c|c|c|c|c|c|}
\hline \multicolumn{7}{|c|}{ Window size: $10 \mathrm{k}$} \\
\hline$(\%) /$ Folds & Fold 1 & Fold 2 & Fold 3 & Fold 4 & Fold 5 & Fold 6 \\
\hline \multicolumn{7}{|c|}{ DeepSNPv1 (no attention) } \\
\hline F1 MAC & 84.64 & 64.58 & 54.74 & 73.75 & 60.28 & 55.85 \\
\hline F1 POS & 71.97 & 37.18 & 22.99 & 53.24 & 40.33 & 27.83 \\
\hline PREC MAC & 81.32 & 61.47 & 56.26 & 68.53 & 62.23 & 57.45 \\
\hline PREC POS & 64.19 & 24.11 & 13.26 & 37.83 & 25.78 & 16.93 \\
\hline REC MAC & 89.04 & 83.61 & 81.40 & 89.80 & 80.07 & 75.75 \\
\hline REC POS & 81.90 & 81.20 & 86.14 & 89.84 & 92.52 & 78.18 \\
\hline \multicolumn{7}{|c|}{ DeepSNPv1 (final attention) } \\
\hline F1 MAC & 80.86 & 68.11 & 58.04 & 70.07 & 63.85 & 59.26 \\
\hline F1 POS & 65.67 & 42.76 & 27.59 & 47.38 & 44.07 & 31.62 \\
\hline PREC MAC & 75.66 & 63.84 & 57.94 & 65.73 & 63.83 & 58.95 \\
\hline PREC POS & 52.40 & 28.61 & 16.13 & 32.38 & 28.97 & 19.82 \\
\hline REC MAC & 90.63 & 86.53 & 87.29 & 87.75 & 82.31 & 78.09 \\
\hline REC POS & 87.93 & 84.62 & 95.05 & 88.28 & 92.06 & 78.18 \\
\hline \multicolumn{7}{|c|}{ DeepSNPv2 (no attention) } \\
\hline F1 MAC & 85.80 & 76.98 & 61.18 & 84.34 & 81.23 & 70.68 \\
\hline F1 POS & 73.53 & 56.25 & 30.33 & 70.92 & 67.08 & 46.84 \\
\hline PREC MAC & 91.17 & 78.18 & 58.89 & 81.70 & 78.46 & 66.75 \\
\hline PREC POS & 85.23 & 58.88 & 18.81 & 64.94 & 59.93 & 35.92 \\
\hline REC MAC & 81.86 & 75.89 & 82.11 & 87.60 & 84.99 & 79.46 \\
\hline REC POS & 64.66 & 53.85 & 78.22 & 78.13 & 76.17 & 67.27 \\
\hline \multicolumn{7}{|c|}{ DeepSNPv2 (final attention) } \\
\hline F1 MAC & 87.26 & 77.64 & 62.63 & 82.16 & 78.65 & 70.32 \\
\hline F1 POS & 76.28 & 57.52 & 32.37 & 67.10 & 63.0 & 46.25 \\
\hline PREC MAC & 91.82 & 78.61 & 59.70 & 78.07 & 75.07 & 66.40 \\
\hline PREC POS & 86.23 & 59.63 & 20.47 & 57.54 & 53.04 & 35.24 \\
\hline REC MAC & 83.74 & 76.75 & 82.40 & 88.18 & 84.61 & 79.34 \\
\hline REC POS & 68.39 & 55.56 & 77.23 & 80.47 & 77.57 & 67.27 \\
\hline
\end{tabular}

All values are shown in $\%$.

results presented in this section were computed using the full-length genomic probes. For details see Section 3.1.

For each fold, we report F1-Score (F1), Precision (PREC), and Recall (REC). For all scores, averaging is applied in two different ways: macro (MAC - calculate metrics for each label and find their unweighted mean) and binary (POS - calculate metrics only for positive labels, i.e., with breakpoint). We evaluate all methods based on the window sizes $(n=[40 k, 20 k, 10 k, 5 k])$ that were used for training. We did not use windows larger than 40k due to computational and memory limits. Two sets of evaluation results are provided, namely Standard Evaluation and Localization Evaluation.

5.1.1. Standard evaluation. Each model is evaluated with the same window size that it is trained with. Thus, each model has to predict the existence of a breakpoint for the whole window.

5.1.2. Localization evaluation. The Localization Unit can provide predictions with higher resolutions than other detection models. For example, DeepSNPv1 trained with 40k windows of probes produces 156 localization outputs. Following, instead of predicting one probability for 40k probes, 156 probabilities are produced (roughly one prediction for each 256). For visualizing the localization output in Figure 3, each probability value in the localization output is repeated 256 times to have the localization output with the same length as the input in the given example above.

To evaluate the localization unit with our evaluation measures, we threshold the localization probabilities by 0.5 and use the outcome as breakpoint predictions with higher resolution. We evaluate the localization 
Table 8. Cross-Validation Results for Baseline Experiments with Window Size 5K

\begin{tabular}{|c|c|c|c|c|c|c|}
\hline \multicolumn{7}{|c|}{ Window size: $5 k$} \\
\hline$(\%) /$ Folds & Fold 1 & Fold 2 & Fold 3 & Fold 4 & Fold 5 & Fold 6 \\
\hline \multicolumn{7}{|l|}{ Rawcopy } \\
\hline F1 MAC & 51.27 & 37.25 & 46.23 & 49.70 & 46.04 & 35.88 \\
\hline F1 POS & 9.68 & 10.02 & 20.96 & 20.54 & 17.68 & 19.97 \\
\hline PREC MAC & 52.47 & 52.46 & 55.66 & 55.72 & 54.85 & 55.29 \\
\hline PREC POS & 5.15 & 5.29 & 11.77 & 11.44 & 9.70 & 11.12 \\
\hline REC MAC & 83.41 & 70.89 & 75.85 & 82.55 & 79.61 & 66.36 \\
\hline REC POS & 80.0 & 94.11 & 95.92 & 100.0 & 100.0 & 97.69 \\
\hline \multicolumn{7}{|l|}{ BLVGG } \\
\hline F1 MAC & 55.66 & 58.92 & 53.70 & 64.70 & 65.14 & 64.97 \\
\hline F1 POS & 11.76 & 19.35 & 10.53 & 32.0 & 32.32 & 33.53 \\
\hline PREC MAC & 74.59 & 75.93 & 66.01 & 67.61 & 76.92 & 80.66 \\
\hline PREC POS & 50.0 & 54.55 & 37.50 & 38.46 & 57.14 & 67.44 \\
\hline REC MAC & 53.30 & 55.73 & 52.75 & 62.71 & 60.90 & 60.71 \\
\hline REC POS & 6.67 & 11.76 & 6.12 & 27.40 & 22.54 & 22.31 \\
\hline \multicolumn{7}{|l|}{ BLDenseNet } \\
\hline F1 MAC & 69.82 & 59.43 & 54.23 & 57.15 & 54.32 & 48.74 \\
\hline F1 POS & 40.0 & 20.93 & 11.32 & 17.24 & 10.67 & 1.50 \\
\hline PREC MAC & 89.67 & 61.59 & 84.77 & 59.72 & 98.02 & 62.85 \\
\hline PREC POS & 80.0 & 25.71 & 75.0 & 23.26 & 100.0 & 33.33 \\
\hline REC MAC & 63.30 & 58.03 & 53.0 & 55.83 & 52.82 & 50.32 \\
\hline REC POS & 26.67 & 17.65 & 6.12 & 13.70 & 5.63 & 0.77 \\
\hline \multicolumn{7}{|c|}{ BLDilated-DenseNet } \\
\hline F1 MAC & 74.70 & 49.17 & 77.47 & 80.83 & 77.84 & 73.99 \\
\hline F1 POS & 50.0 & 13.70 & 57.45 & 63.69 & 57.36 & 51.94 \\
\hline PREC MAC & 69.85 & 53.15 & 78.63 & 76.38 & 80.86 & 74.16 \\
\hline PREC POS & 40.0 & 7.61 & 60.0 & 53.77 & 63.79 & 52.34 \\
\hline REC MAC & 82.89 & 71.36 & 76.42 & 87.53 & 75.41 & 73.82 \\
\hline REC POS & 66.67 & 68.63 & 55.10 & 78.08 & 52.11 & 51.54 \\
\hline \multicolumn{7}{|c|}{ BLLSTM-DenseNet } \\
\hline F1 MAC & 77.92 & 57.19 & 80.30 & 87.45 & 79.40 & 74.67 \\
\hline F1 POS & 56.25 & 22.15 & 62.42 & 75.91 & 60.18 & 52.43 \\
\hline PREC MAC & 76.29 & 56.05 & 90.03 & 89.98 & 89.35 & 83.17 \\
\hline PREC POS & 52.94 & 13.28 & 83.05 & 81.25 & 80.95 & 71.05 \\
\hline REC MAC & 79.76 & 76.57 & 74.69 & 85.25 & 73.70 & 70.07 \\
\hline REC POS & 60.0 & 66.67 & 50.0 & 71.23 & 47.89 & 41.54 \\
\hline
\end{tabular}

All values are shown in \%.

outputs with smaller windows than the window size the model was trained with, for example, localization results for a model trained with $40 \mathrm{k}$ are evaluated based on ground truth of $20 \mathrm{k}, 10 \mathrm{k}$, and $5 \mathrm{k}$ windows. All details about the localization output and the evaluations are provided in Table 1.

\subsection{Baselines}

This section details the Baseline methods (BLM). We used two state-of-the-art feed-forward DNNs, namely VGG (Simonyan and Zisserman, 2014) and DenseNet (Huang et al., 2016). As discussed before, dilated convolution (Gupta and Rush, 2017) as well as LSTMs (Gupta and Rush, 2017) has shown promising results on various tasks on genomic data. Therefore, we adapt DenseNet using dilated convolution (BLDilated-DenseNet), and LSTMs (BLLSTM-DenseNet) for two additional BLMs. Both BLDilated-DenseNet and BLLSTMDenseNet have similarities to DeepSNP: BLDilated-DenseNet has a fully dilated convolution architecture, but without any LSTM layers. BLLSTM-DenseNet has no dilated convolution, but incorporates a bidirectional LSTM layer, which makes it a counterpart of DeepSNPv2 without Localization and Attention Units. 
Table 9. Cross-Validation Results for DeepSNP Experiments with Window Size 5K

\begin{tabular}{|c|c|c|c|c|c|c|}
\hline \multicolumn{7}{|c|}{ Window size: $5 k$} \\
\hline$(\%) /$ Folds & Fold 1 & Fold 2 & Fold 3 & Fold 4 & Fold 5 & Fold 6 \\
\hline \multicolumn{7}{|c|}{ DeepSNPv1 (no attention) } \\
\hline F1 MAC & 77.08 & 59.49 & 53.83 & 49.56 & 63.27 & 46.02 \\
\hline F1 POS & 56.96 & 26.08 & 17.79 & 18.0 & 37.39 & 14.14 \\
\hline PREC MAC & 71.47 & 57.46 & 54.76 & 54.90 & 61.39 & 53.43 \\
\hline PREC POS & 43.81 & 15.40 & 9.92 & 9.91 & 23.67 & 7.72 \\
\hline REC MAC & 88.36 & 86.12 & 83.98 & 83.04 & 85.0 & 74.31 \\
\hline REC POS & 81.40 & 85.12 & 86.09 & 97.79 & 88.98 & 84.48 \\
\hline \multicolumn{7}{|c|}{ DeepSNPv1 (final attention) } \\
\hline F1 MAC & 71.49 & 60.83 & 51.80 & 64.45 & 66.96 & 54.50 \\
\hline F1 POS & 47.39 & 27.94 & 16.10 & 34.89 & 42.47 & 20.86 \\
\hline PREC MAC & 66.04 & 58.13 & 54.29 & 60.67 & 63.52 & 55.55 \\
\hline PREC POS & 32.77 & 16.78 & 8.82 & 21.93 & 27.93 & 12.01 \\
\hline REC MAC & 88.83 & 86.03 & 85.04 & 87.23 & 86.72 & 79.34 \\
\hline REC POS & 85.53 & 83.47 & 92.17 & 85.29 & 88.57 & 79.31 \\
\hline \multicolumn{7}{|c|}{ DeepSNPv2 (no attention) } \\
\hline F1 MAC & 84.02 & 68.50 & 59.59 & 82.97 & 79.45 & 65.05 \\
\hline F1 POS & 69.23 & 38.60 & 24.51 & 67.14 & 61.68 & 34.31 \\
\hline PREC MAC & 89.61 & 69.69 & 57.04 & 82.09 & 77.35 & 61.24 \\
\hline PREC POS & 80.97 & 41.12 & 14.80 & 65.28 & 56.90 & 23.97 \\
\hline REC MAC & 79.91 & 67.46 & 80.90 & 83.90 & 81.99 & 76.78 \\
\hline REC POS & 60.47 & 36.36 & 71.30 & 69.12 & 67.35 & 60.34 \\
\hline \multicolumn{7}{|c|}{ DeepSNPv2 (final attention) } \\
\hline F1 MAC & 84.50 & 69.45 & 59.48 & 83.67 & 79.93 & 67.0 \\
\hline F1 POS & 70.18 & 40.37 & 24.29 & 68.53 & 62.64 & 37.63 \\
\hline PREC MAC & 89.56 & 71.81 & 56.96 & 82.17 & 77.39 & 62.94 \\
\hline PREC POS & 80.81 & 45.36 & 14.67 & 65.33 & 56.81 & 27.34 \\
\hline REC MAC & 80.68 & 67.58 & 80.47 & 85.35 & 83.14 & 77.33 \\
\hline REC POS & 62.02 & 36.36 & 70.43 & 72.06 & 69.80 & 60.34 \\
\hline
\end{tabular}

All values are shown in $\%$.

The architectures of the implemented BLVGG, BLDenseNet, and BLDilated-DenseNet are shown in Table 10, and the architecture of the BLLSTM-DenseNet is shown in Table 11.

More details about the network architectures can be found in our source code. ${ }^{2}$

All baselines were trained for 200 epochs with a batch size of 25 using AMSGrad (Reddi et al., 2018) (an adaptive version of SGD), categorical cross-entropy loss, initial learning rate of 0.001 , which was reduced on plateau by 0.5 . The patience of 33 and early stopping of 100 epochs were carried out as well.

DeepSNP was trained with the same hyperparameters, optimizer, and training strategy as the BLM. The architecture of DeepSNP can be found in Table 12. DeepSNP and DL BLM are implemented in Keras (Chollet et al., 2015) using the TensorFlow (Abadi et al., 2016) backend and were trained on a NVIDIA DGX Station.

We also compared our results with breakpoints detected by Rawcopy, a specialized tool for CNV detection. Rawcopy data processing was done according to Section 3. Rawcopy uses various built-in reference data precompiled from a large number [2875 samples, supplementary table 1 of Mayrhofer et al. (2016)] of ethnically diverse samples, with variations also in technical quality to improve LRR and BAF normalization. Therefore, in the sense of training data, Rawcopy benefited from significantly more data compared with DeepSNP and the rest of the DL BLM that were only trained on a subset of the 50 available samples. To evaluate Rawcopy for breakpoint prediction, first, the CNSs were predicted for each probe and then converted to our binary format as explained in Section 3.1.

\footnotetext{
${ }^{2}$ https://github.com/eghbalz/deepsnp.
} 
Table 10. Baseline Feed-Forward architectures:

BLVGG, BLDenseNet, AND BLDilated-DenseNet

\begin{tabular}{l}
\multicolumn{1}{c}{$B L V G G$} \\
\hline Input $2 \times 40 k \times 1$ \\
$1 \times 10$ Conv(stride- $1 \times 3)-32-$ BN-ReLu \\
$1 \times 5$ Conv(stride- $1 \times 1)-64-$ ReLu \\
$1 \times 5$ Conv(stride- $1 \times 1)-64-$ BN-ReLu \\
$1 \times 2$ Max-Pooling(stride- $1 \times 2)+$ Drop-Out $(0.4)$ \\
$1 \times 3$ Conv(stride- $1 \times 1)-128-$ ReLu \\
$1 \times 3$ Conv(stride- $1 \times 1)-128-$ BN-ReLu \\
$1 \times 2$ Max-Pooling(stride- $1 \times 2)+$ Drop-Out $(0.3)$ \\
$1 \times 3$ Conv(stride- $1 \times 1)-256-$ ReLu \\
$1 \times 3$ Conv(stride- $1 \times 1)-256-$ ReLu \\
$1 \times 3$ Conv(stride- $1 \times 1)-256-$ BN-ReLu \\
$1 \times 2$ Max-Pooling(stride- $1 \times 2)+$ Drop-Out $(0.2)$ \\
$1 \times 3$ Conv(stride- $1 \times 1)-512-$ ReLu \\
$1 \times 3$ Conv(stride- $1 \times 1)-512-$ ReLu \\
$1 \times 3$ Conv(stride- $1 \times 1)-512-$ BN-ReLu \\
$1 \times 2$ Max-Pooling(stride- $1 \times 2)+$ Drop-Out $(0.2)$ \\
$1 \times 3$ Conv(stride- $1 \times 1)-512-$ ReLu \\
$1 \times 3$ Conv(stride- $1 \times 1)-512-$ ReLu \\
$1 \times 3$ Conv(stride- $1 \times 1)-512-$ BN-ReLu \\
$1 \times 2$ Max-Pooling(stride- $1 \times 2)+$ Drop-Out $(0.2)$ \\
$1 \times 1$ Conv(stride- $1 \times 1)-2-$ ReLu \\
Global-average-pooling \\
\hline $2-$ Way softmax \\
\hline
\end{tabular}

Input $2 \times 40 k \times 1$

$1 \times 10$ ConvBn(stride- $1 \times 5)-208+$ Drop-Out $(0.2)$

Feature learning layers (Table 13)

$1 \times 1$ Conv (stride- $1 \times 1)-2-\operatorname{ReLu}$

Global-average-pooling

2-Way softmax

BLDilated-DenseNet

Input $2 \times 40 k \times 1$

$1 \times 10$ DilConvBN(dilation-1 $\times 3$ )-208+Drop-Out(0.2)

Feature learning layers with dilation (Table 13)

$1 \times 1$ Conv (stride- $1 \times 1)-2-\operatorname{ReLu}$

Global-average-pooling

2-Way softmax

BN, batch normalization; Conv, 2D convolution layer; ConvBN, 2D convolution layer with batch normalization and ReLu activation; ReLu, rectified linear unit.

\subsection{Results and Discussions}

The results for each evaluated algorithm and window size combination are shown in Tables 2, 4, 6, 8 (BLM) and Tables 3, 5, 7, 9 (DeepSNP). For a better visual comparison, we provide these results in bar plots grouped by window size in Figures 4-6.

The evaluation results of DeepSNP (v1 and v2) localization output are provided in Figures 7-9 based on 5k windows, Figures 10-12 based on 10k windows, and Figures 13-15 based on 20k windows. 
Table 11. Baseline Recurrent Neural Network Architectures:

BLLSTM-DENSENET

\begin{tabular}{l}
\hline \multicolumn{1}{c}{ BLLSTM-DenseNet } \\
\hline Input $2 \times 40 k \times 1$ \\
\hline $1 \times 10$ ConvBN(stride-1 $\times 5)-208+$ Drop-Out $(0.2)$ \\
Feature learning layers without dilation (Table 13) \\
Bidirectional LSTM-256 \\
$1 \times 1$ Conv(stride-1 $\times 1)-2-$ ReLu \\
Global-average-pooling \\
2-Way softmax \\
\hline
\end{tabular}

5.3.1. Standard evaluation. In this section, we discuss the standard evaluation of all methods trained and evaluated on different window sizes in terms of MAC and POS F1, Precision, and Recall.

\subsubsection{F1}

Figure 4 shows that the best results have been achieved using DeepSNPs with 40k window size (both MAC - also takes the negative class ${ }^{3}$ into account and POS - only focuses on the availability of a breakpoint). BLDilated-DenseNet and BLLSTM-DenseNet achieved the second-best results (MAC and POS) with 40k window size. Rawcopy, BLVGG, and BLDenseNet achieved the lowest performance among all methods for both F1 measures with 40k window size. Among DL methods, there is a significant performance gap between the designed architectures (DeepSNPs, BLLSTM-DenseNet, BLDilatedDenseNet) and the two unmodified baselines (BLVGG and BLDenseNet), which suggests that our architecture modifications significantly improved the performance of original architectures. As the window sizes start to shrink, the performance of DeepSNPs, BLLSTM-DenseNet, and BLDilated-DenseNet starts to degrade. This is not surprising as they are designed to take advantage of long sequences. ${ }^{4}$ In contrast, the performance of BLVGG and BLDenseNet does not improve by using larger window sizes, suggesting that these architectures are not suitable for processing very long sequential data and require modifications.

Comparing DeepSNPs, DeepSNPv2 performs better than DeepSNPv1 when dealing with smaller windows. This can be explained by the fact that as DeepSNPv2 does not incorporate dilated convolutions, it has smaller receptive fields and therefore can better deal with smaller inputs. For larger windows, it can achieve comparable performances with DeepSNPv1. Comparing DeepSNPs with and without Attention Unit, it can be seen that while these two models perform similarly in terms of precision, the Final Attention model consistently provides results with a smaller standard deviation (STD), which suggests that adding the Final Attention Unit provides more stable results and less variance in predictions.

\subsubsection{Precision}

Figure 5 reveals that similarly to F1, DeepSNPs achieve the best results for 40k windows (MAC and POS).

Again, BLDilated-DenseNet and BLLSTM-DenseNet achieved the second-best and Rawcopy, BLDenseNet, and BLVGG yielded the worst results. As observed in F1, with shrinking window size, the performance of DeepSNPs, BLLSTM-DenseNet, and BLDilated-DenseNet starts to degrade. The performance of BLVGG and BLDenseNet does not improve by using larger window sizes. A similar significant performance gap between (DeepSNPs, BLLSTM-DenseNet and BLDilated-DenseNet) and (Rawcopy, BLDenseNet and BLVGG.) can be observed in MAC and POS Precision. Also, DeepSNP with attention model consistently provides results with smaller STD similar to F1 results.

\subsubsection{Recall}

Figure 6 shows that unlike F1 and Precision, Recall is not significantly effected by the window size, which can be a side effect of having a low number of positive samples compared with negative samples. A

\footnotetext{
${ }^{3}$ The number of windows with breakpoints in each genomic sample is around 5\% of the number of windows without breakpoints.

${ }^{4}$ Both LSTM and dilated convolution can deal with long sequences to model long-term relationships between probes.
} 
Table 12. DeepSNP architecture

\begin{tabular}{ll}
\hline \multicolumn{1}{c}{ DeepSNP } \\
\hline Input $2 \times 40 k \times 1$ & \\
\hline $1 \times 10$ DilConvBN(dilation-1 $\times 5)-208+$ Drop-Out $(0.2)$ & \\
Feature learning layers (Table 13) & Softmax (no-attention) \\
Bidirectional GRU-256 & \\
Final attention layer & \\
Averaging layer & \\
\hline GRU, gated recurrent unit/layer.
\end{tabular}

GRU, gated recurrent unit/layer.

similar pattern can be seen in MAC Recall: best results are achieved by DeepSNPs, followed by BLDilatedDenseNet and BLLSTM-DenseNet; the lowest results are from BLDenseNet and BLVGG. Unlike in F1 and Precision, Rawcopy performs slightly better than BLDenseNet and BLVGG in terms of MAC Recall. Looking at POS Recall, it can be observed that Rawcopy is better than all other methods, followed by

Table 13. Feature Learning Architectures for DeepSNP and BLDenseNet

\begin{tabular}{|c|c|c|}
\hline & DeepSNP feature learning / BLDilated-DenseNet & BLDenseNet \\
\hline Input & $2 \times 40 k \times 1$ & \\
\hline Conv & {$[1 \times 5$ DConv: $1 \times 2 / 1 \times 9]-16$} & [ $1 \times 5$ Conv stride: $1 \times 2]-16$ \\
\hline $\mathrm{BN}$ & $\mathrm{BN}$ & \\
\hline DenseB (1) & {$\left[\begin{array}{l}{[1 \times 3 \text { Conv }]-12} \\
\text { concat input }\end{array}\right.$} & \\
\hline TransL (1) & $\begin{array}{l}{[1 \times 5 \text { DConv: } 1 \times 2 / 1 \times 27]-64} \\
1 \times 2 \text { average pool } 1 \times 3 \text {, stride } 1 \times 2 \\
\text { BN }\end{array}$ & [ $1 \times 5$ Conv stride: $1 \times 1]-64$ \\
\hline DenseB (2) & {$\left[\begin{array}{l}{[1 \times 3 \text { Conv }]-12} \\
\text { concat input }\end{array}\right.$} & \\
\hline TransL (2) & $\begin{array}{l}{[1 \times 5 \text { DConv } 1 \times 2 / 1 \times 81]-112} \\
1 \times 2 \text { average pool stride } 1 \times 2 \\
\text { BN }\end{array}$ & [ $1 \times 5$ Conv stride: $1 \times 2]-112$ \\
\hline DenseB (3) & {$\left[\begin{array}{l}{[1 \times 3 \text { Conv }]-12} \\
\text { concat input }\end{array}\right.$} & \\
\hline TransL (3) & $\begin{array}{l}{[1 \times 2 \text { Conv stride } 1 \times 2]-160} \\
1 \times 2 \text { average pool stride } 2 \\
\text { BN }\end{array}$ & \\
\hline DenseB (4) & {$\left[\begin{array}{l}{[1 \times 3 \text { Conv }]-12} \\
\text { concat input }\end{array}\right] \times 4$} & \\
\hline TransL (4) & $\begin{array}{l}{[1 \times 2 \text { Conv, stride } 1 \times 2]-208} \\
1 \times 2 \text { average pool stride } 2 \\
\text { BN }\end{array}$ & \\
\hline DenseB (5) & {$\left[\begin{array}{l}{[1 \times 3 \text { Conv }]-12} \\
\text { concat input }\end{array}\right] \times 4$} & \\
\hline TransL (5) & $\begin{array}{l}{[1 \times 2 \text { Conv stride } 1 \times 2]-256} \\
1 \times 2 \text { average pool stride } 2 \\
\text { BN }\end{array}$ & \\
\hline DenseB (6) & {$\left[\begin{array}{l}{[1 \times 3 \text { Conv }]-12} \\
\text { concat input }\end{array}\right] \times 4$} & \\
\hline
\end{tabular}

DConv, 2D dilated convolution layer; DenseB, dense block; TransL, transition layer. 

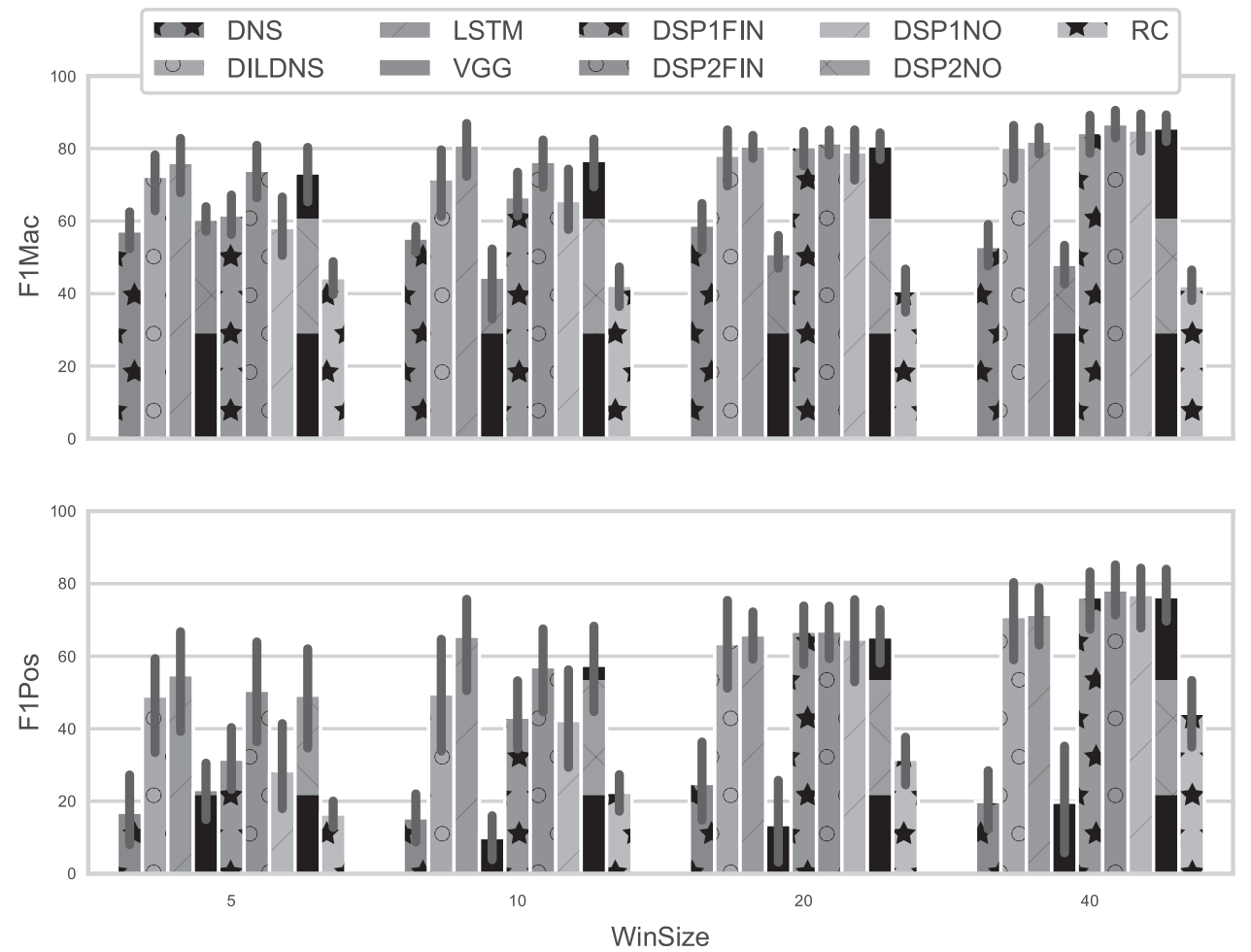

FIG. 4. F1 of different methods, grouped by window size (STD is computed over different folds). STD, standard deviation.
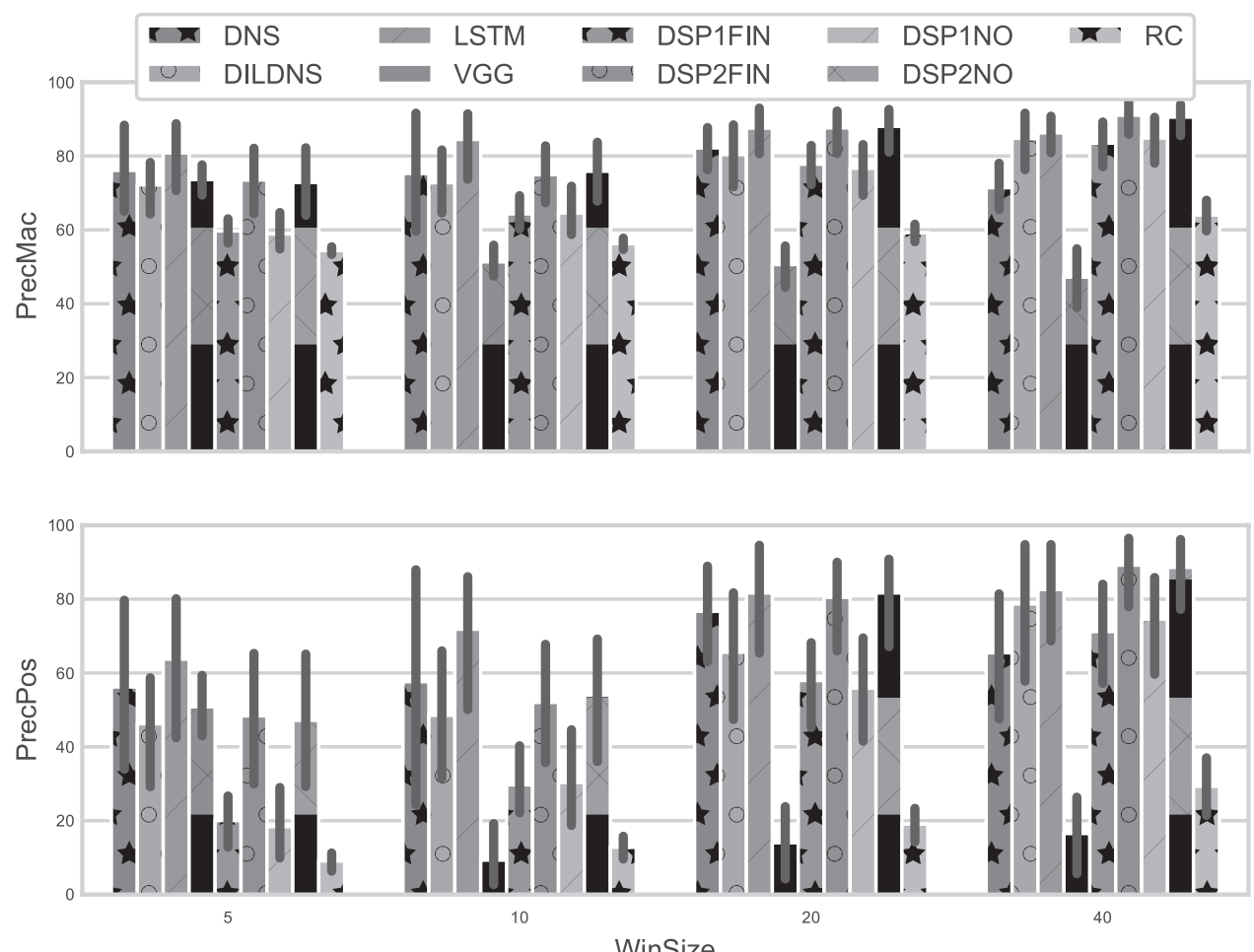

FIG. 5. Precision of different methods, grouped by window size (STD is computed over different folds). 

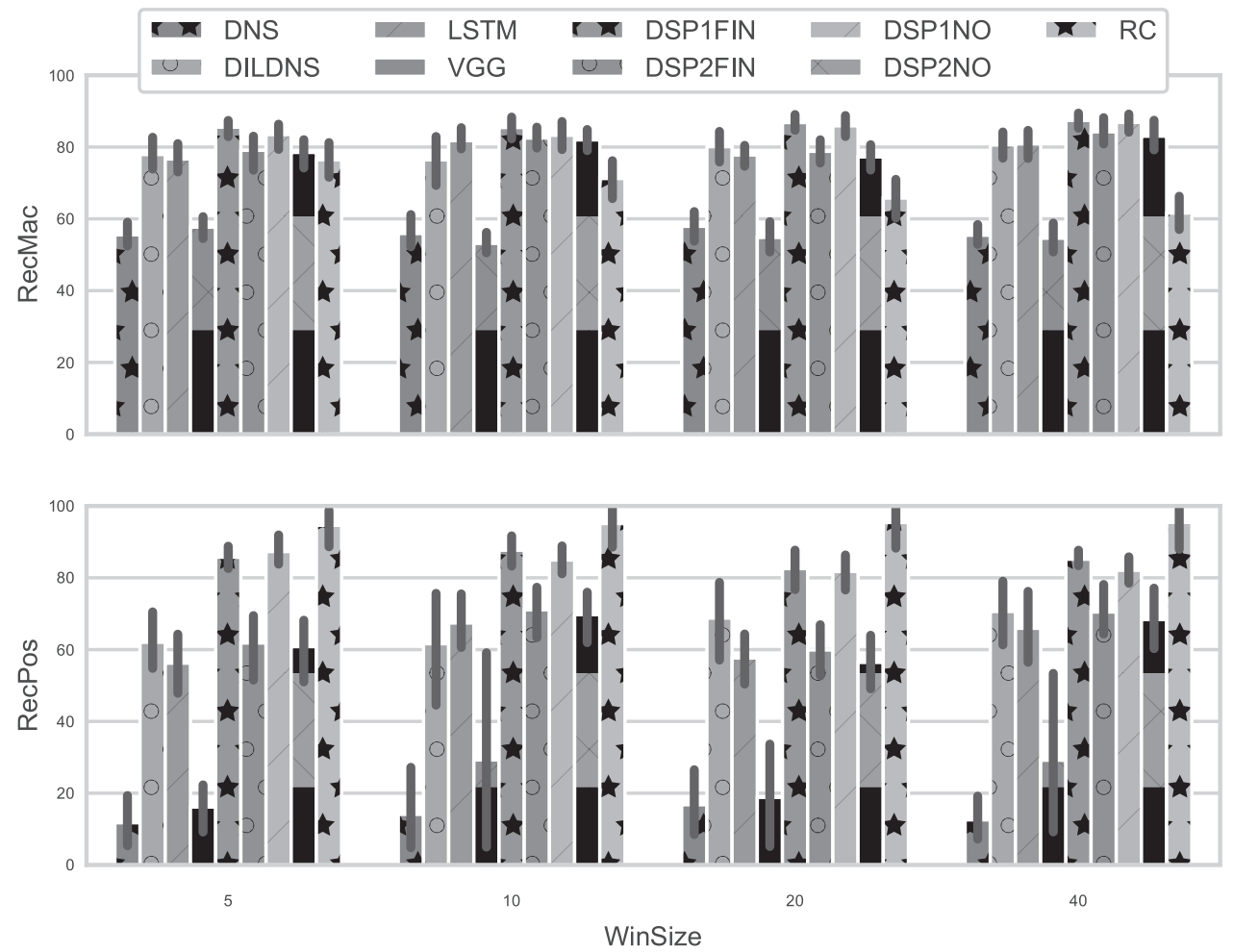

FIG. 6. Recall of different methods, grouped by window size (STD is computed over different folds).

DeepSNPv1, DeepSNPv2, BLDilated-DenseNet, and BLLSTM-DenseNet. BLDenseNet and BLVGG achieved the lowest results. This is understandable as Rawcopy is specifically built to have high precision in detecting breakpoints.

Unlike in F1 and Precision results, it can be seen that DeepSNPv1 performs better than DeepSNPv2 in terms of Recall in all window sizes. One of the benefits of using dilated convolution is that we can have activations
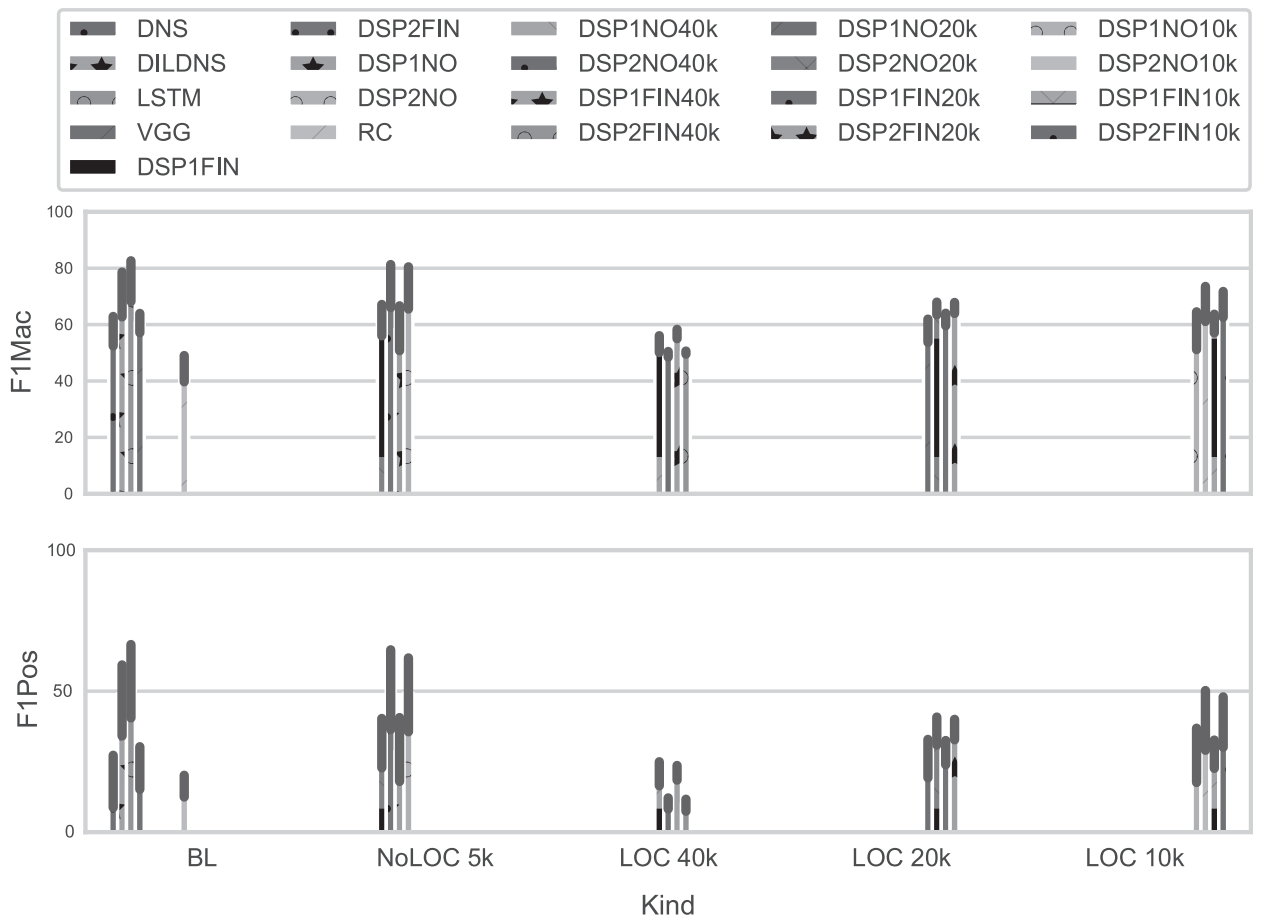

FIG. 7. F1 of localization and all other methods (5k win). (STD is computed over different folds). 

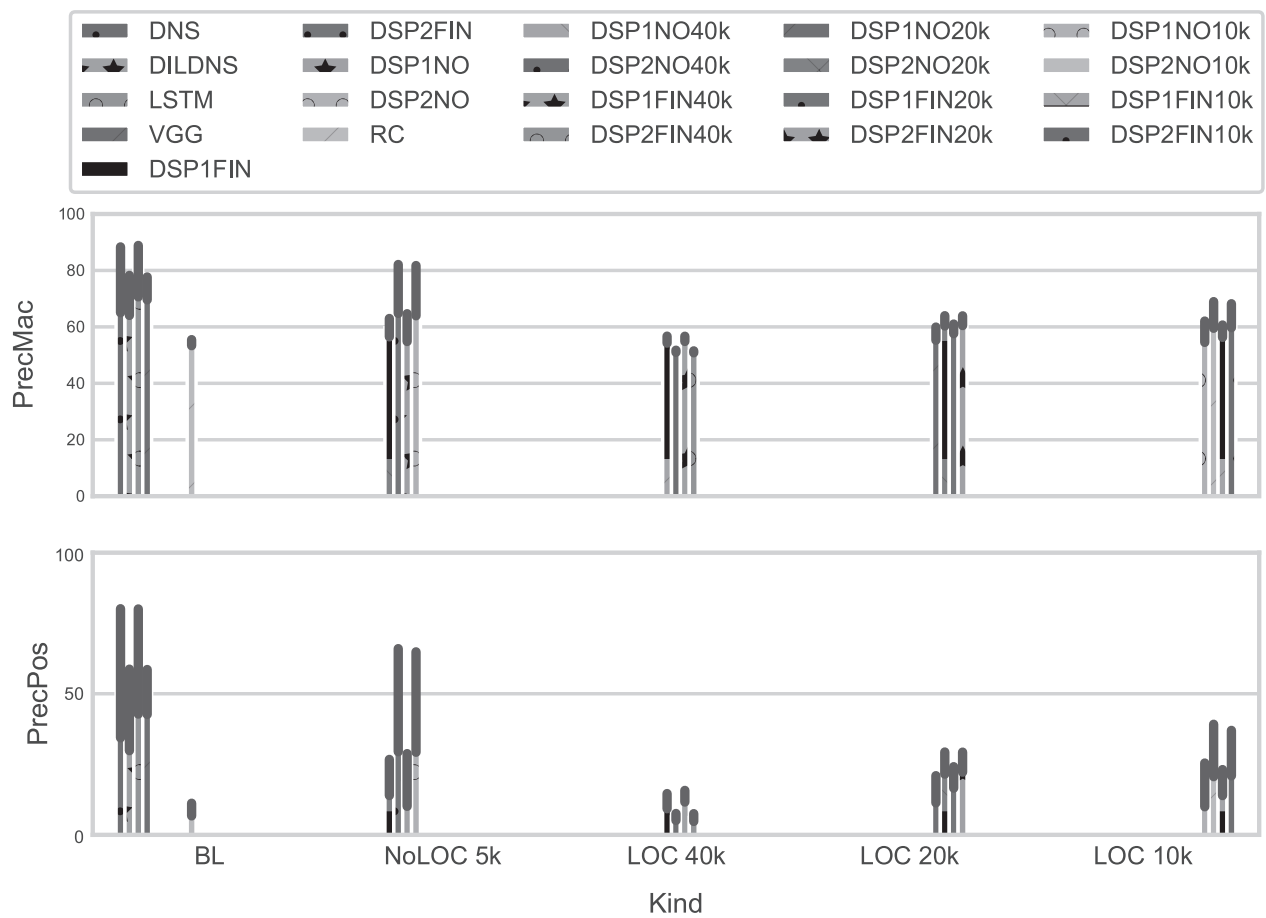

FIG. 8. Precision of localization and all other methods (5k win). (STD is computed over different folds).

with higher dimensions in the upper layers of our neural network. This is desired when we want to have a higher resolution of activations: for example, for the case of 40k, using dilated convolution in DeepSNPv1 results in 156 activations in the localization layer, while in DeepSNPv2, with no dilation this dimensionality is only 8 . This means that the network produces at least one activation for every 256 probes in the input in DeepSNPv1, while the model creates one activation only for every $5 \mathrm{k}$ probes in the input. Therefore, the former results in discovering more breakpoints, and hence, higher POS Recall. It appears that not only DeepSNP with Final

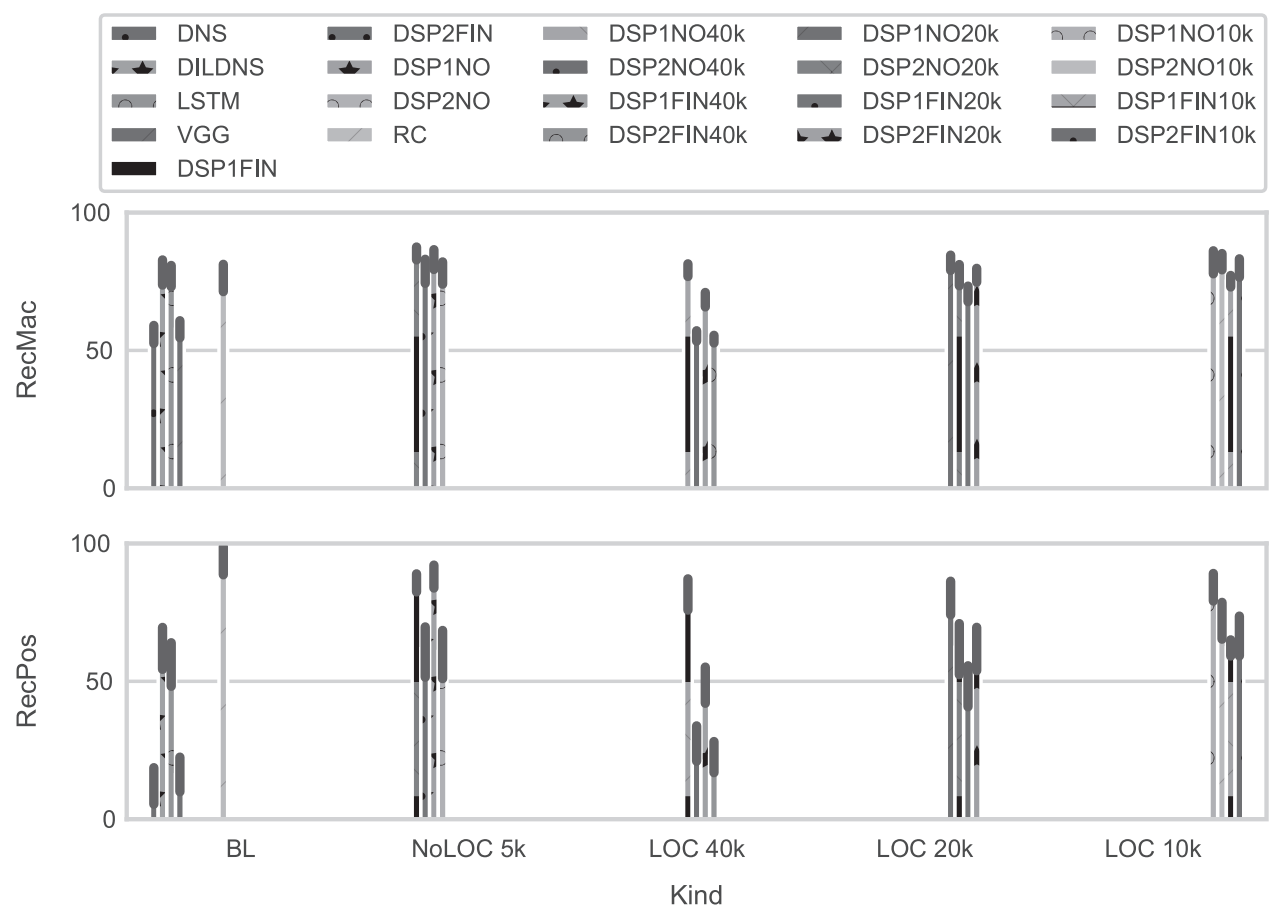

FIG. 9. Recall of localization and all other methods (5k win). (STD is computed over different folds). 


\begin{tabular}{|c|c|c|c|c|c|c|c|c|c|}
\hline 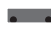 & DNS & - & DSP1FIN & $\cdot$. & $\mathrm{RC}$ & 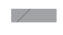 & DSP1FIN40k & $n$ & DSP2NO2Ok \\
\hline 1 & DILDNS & 1 & DSP2FIN & 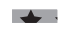 & DSP1NO40k & .. & DSP2FIN40k & 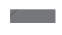 & DSP1FIN20k \\
\hline n & LSTM & $n=$ & DSP1NO & $=0$ & DSP2NO40k & 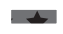 & DSP1NO20k & . & DSP2FIN20k \\
\hline 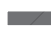 & VGG & $\gamma$ & DSP2NO & & & & & & \\
\hline
\end{tabular}
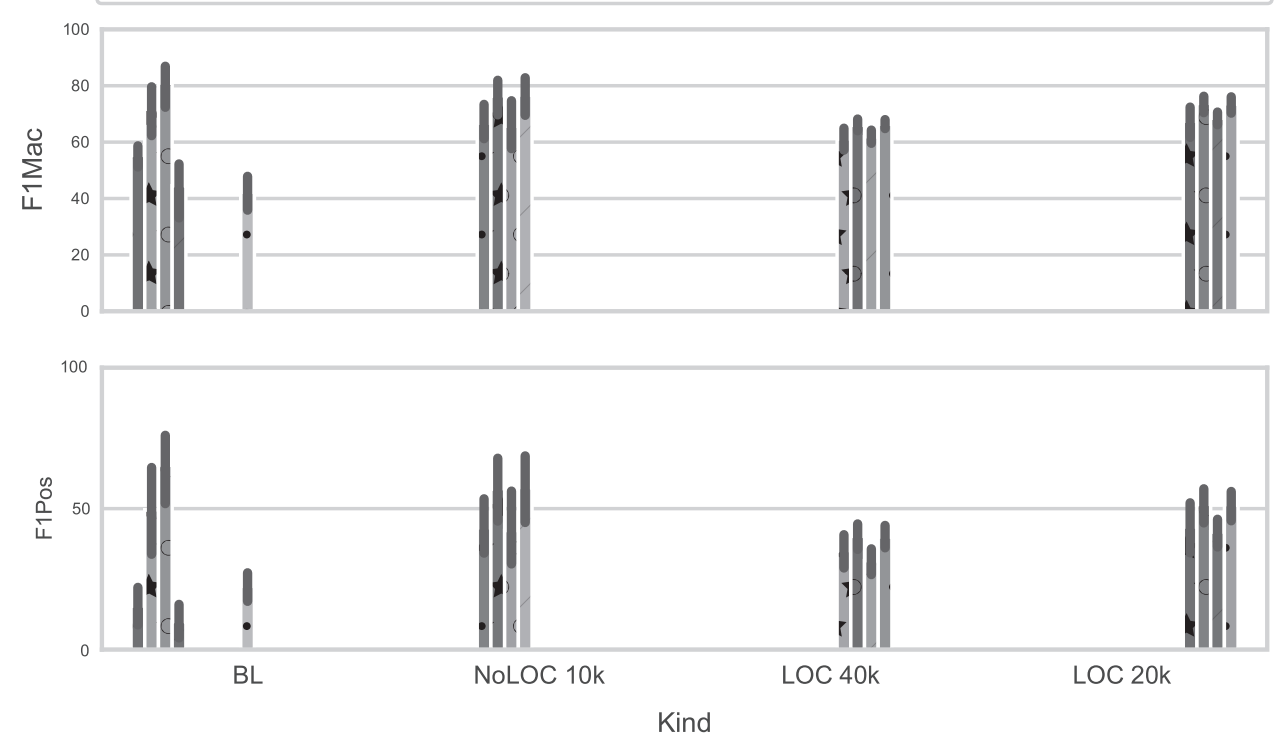

FIG. 10. F1 of localization and all other methods (10k win). (STD is computed over different folds).

Attention Unit provides performances with smaller STD but also it achieves slightly higher Recall compared with the No Attention model, especially for positive Recall with higher windows.

5.3.2. Localization evaluation. In this section, we discuss the evaluation results of the Localization Unit for DeepSNPv1 and DeepSNPv2 trained on window sizes of 40k, 20k, and 10k. Each respective model is evaluated on all smaller window sizes than it was trained with. To put these results in perspective, we

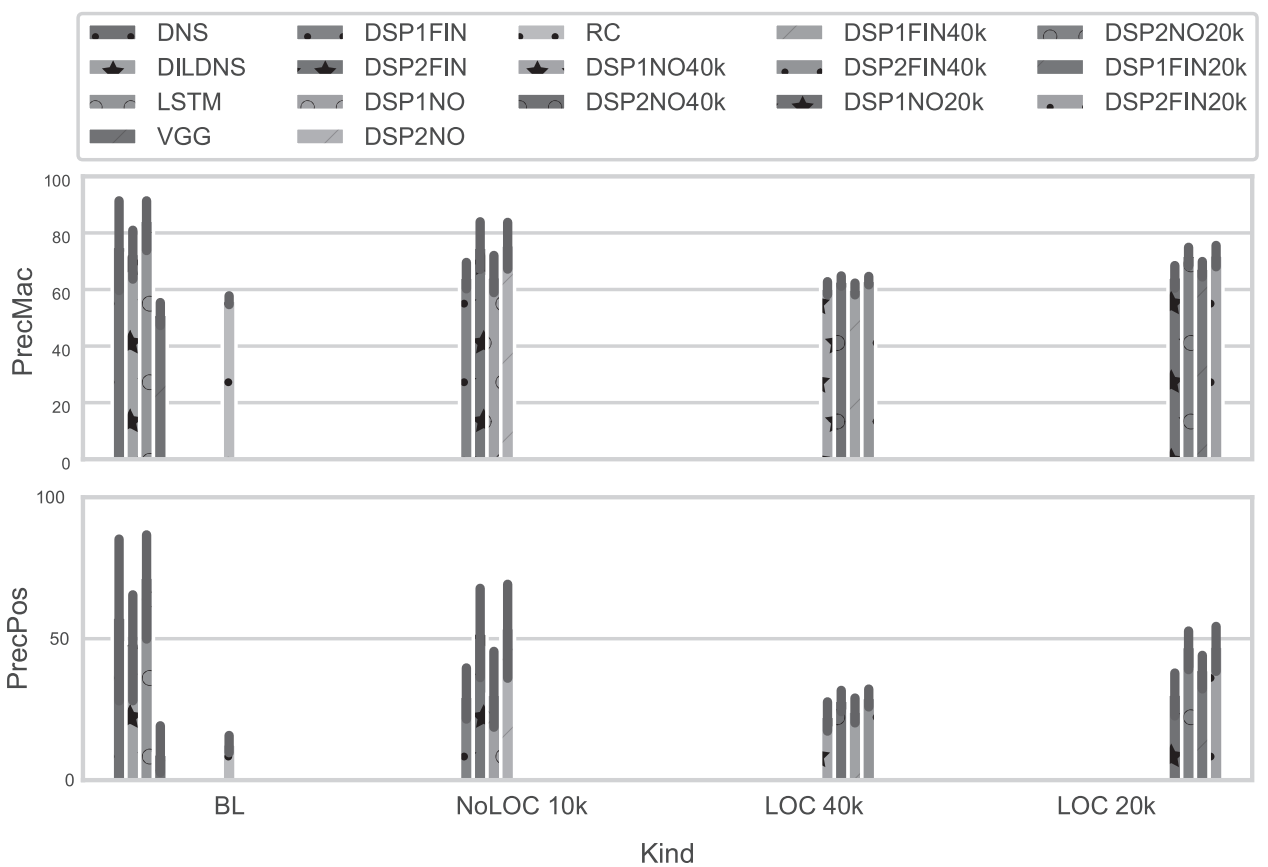

FIG. 11. Precision of localization and all other methods (10k win). (STD is computed over different folds). 

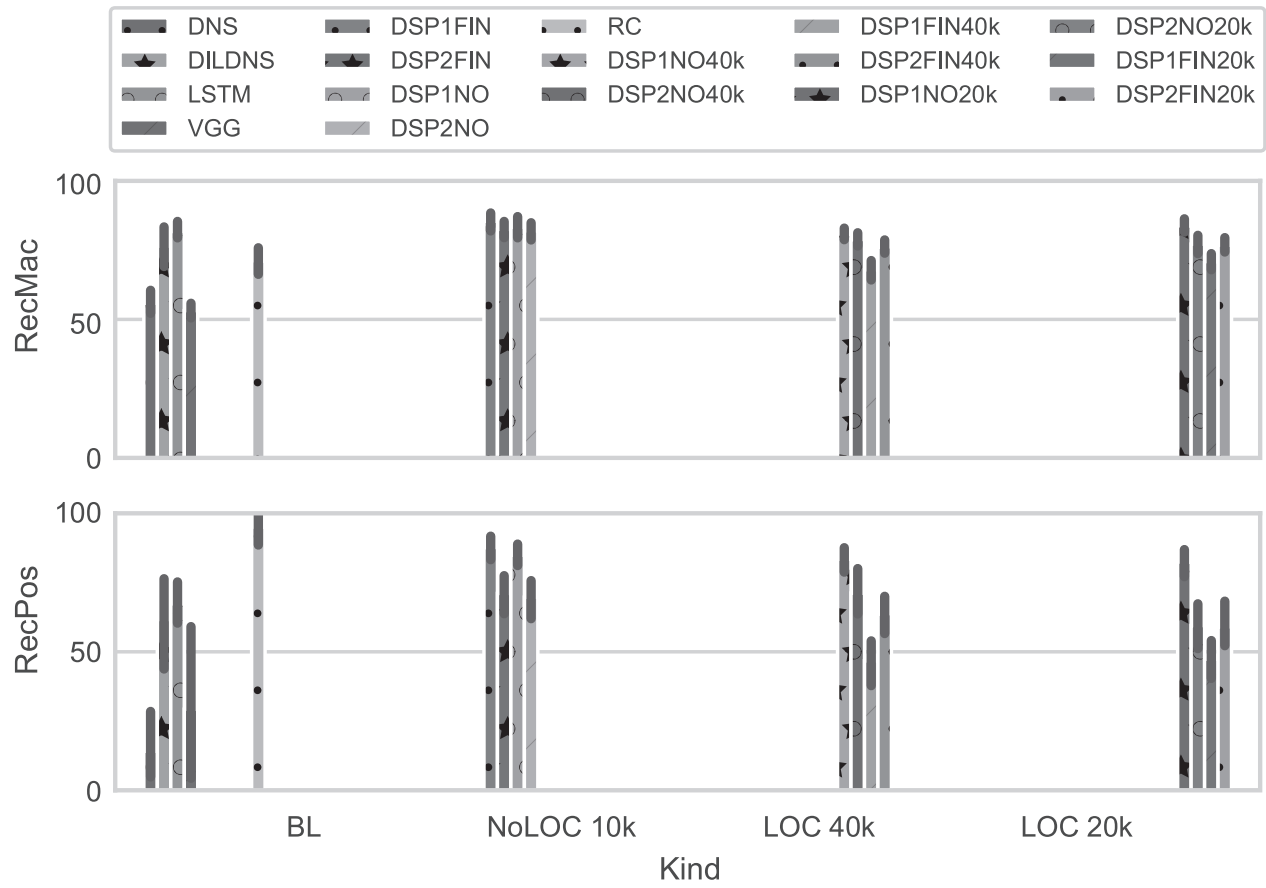

FIG. 12. Recall of localization and all other methods (10k win). (STD is computed over different folds).

also provide the results from the final predictions (not localization outputs) for BLMs ( $x$-axis: $B L$ ) as well as DeepSNPs ( $x$-axis: NoLOC $\{m\} k$ label where $m$ is the window size we both trained and evaluated with). Evaluation results can be found over the LOC $\{n\} k$ label in the figure legends where $n$ is the window size we evaluated the localization output with, while their models were trained with window size $m$. Table 1 provides information about which localization outputs were evaluated with which window size.

The models labeled BL and NoLOC are trained with window size 5k. Localization results from DeepSNPs are trained with 40k, 20k, and 10k and can be found under $\operatorname{LOC}\{n\} k(n=40,20,10)$.

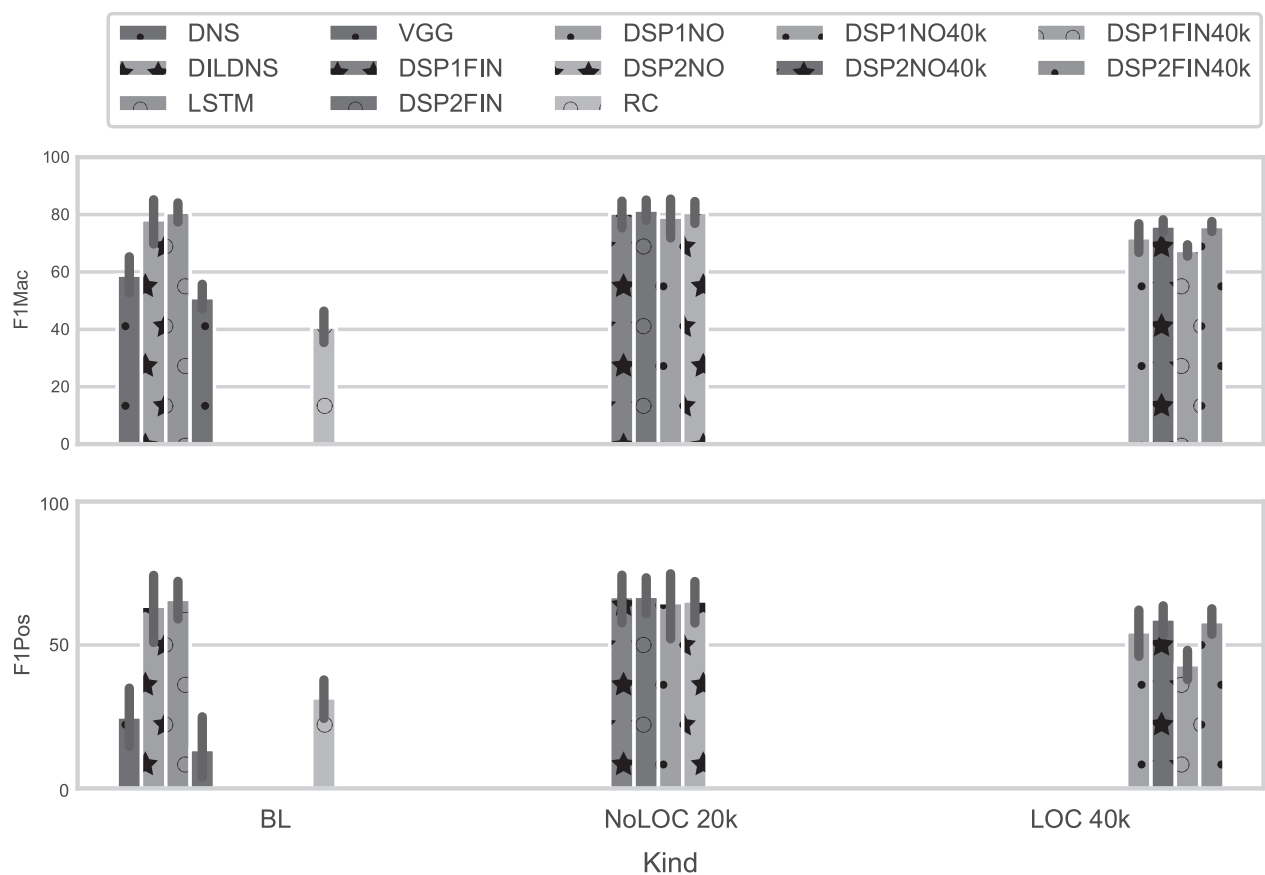

FIG. 13. F1 of localization and all other methods (20k win). (STD is computed over different folds). 

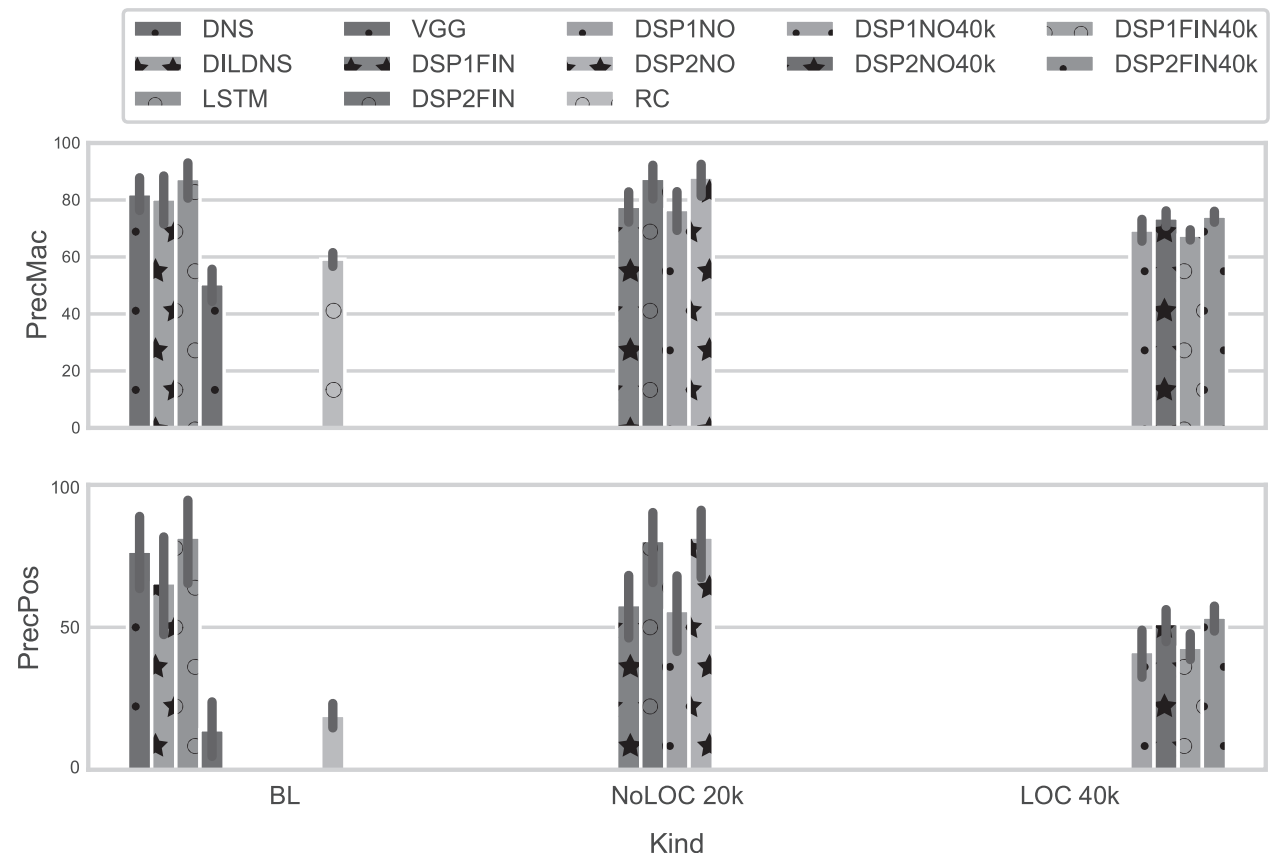

FIG. 14. Precision of localization and all other methods (20k win). (STD is computed over different folds).

\subsubsection{F1}

As seen in Figure 7, localizations from DeepSNPs trained with 10k and 20k have comparable results with the best models trained with 5k. Models trained with 20k achieve predictions with smaller STDs compared with 10k models. F1 of localizations starts to degrade as the gap between the training window and evaluation window increases. It can also be seen that DeepSNPv2 provides more accurate localizations when trained with smaller windows, while DeepSNPv1 is more accurate when trained with larger windows.

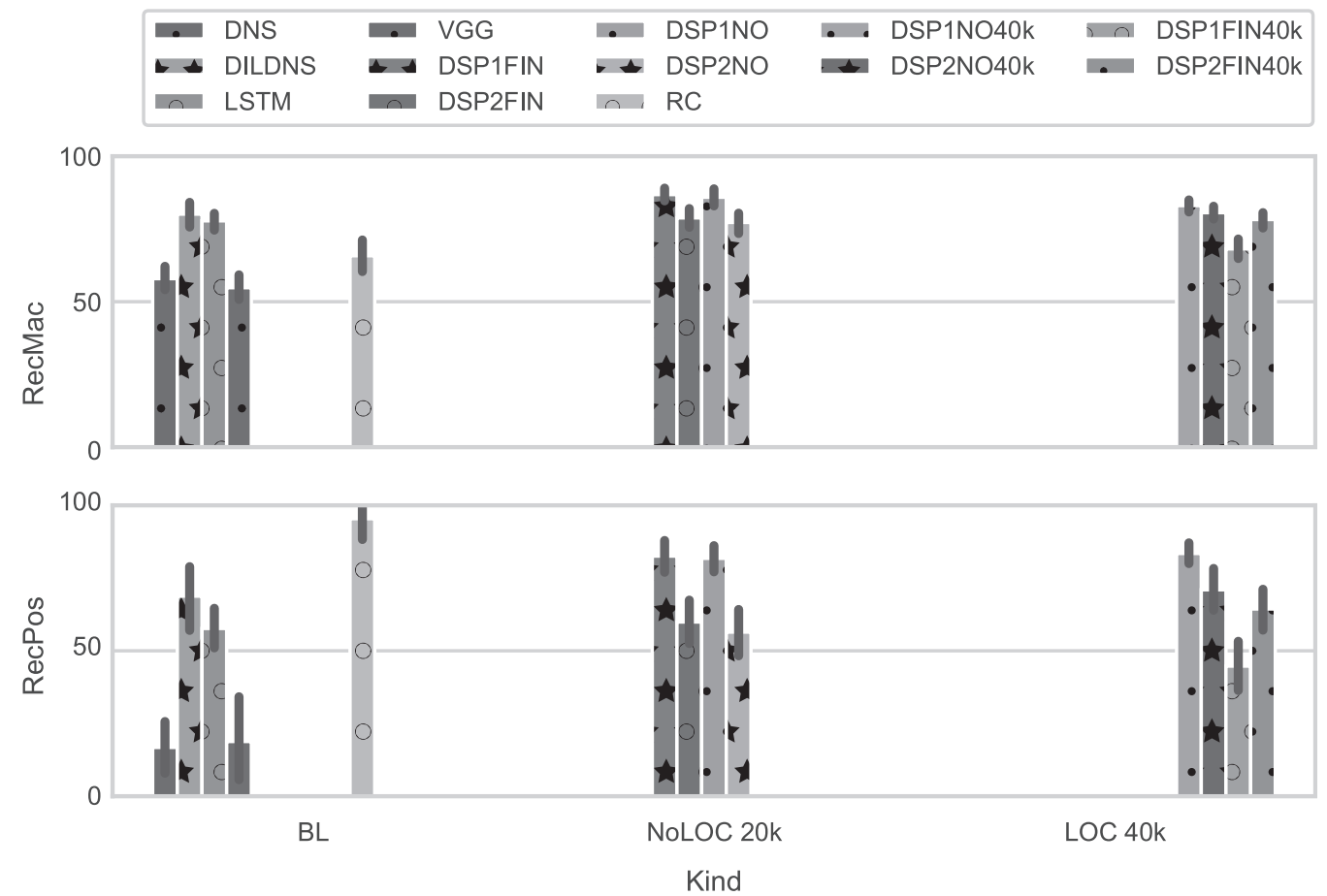

FIG. 15. Recall of localization and all other methods (20k win). (STD is computed over different folds). 
These results are also aligned with our standard evaluations and can be similarly explained. In addition, it seems that the STD of F1 from the Final Attention model is lower than the No Attention model, which is in line with the standard evaluation results.

\subsubsection{Precision}

As shown in Figure 8, DeepSNP localizations trained with 10k and 20k have comparable results with the best models trained with $5 \mathrm{k}$. If trained with $20 \mathrm{k}$, smaller STDs are observed compared with $10 \mathrm{k}$ models. The Precision of localizations starts to degrade as the gap between training and evaluation window increases (especially from 10k to 5k). DeepSNPv1 performs better for 40k models, while DeepSNPv2 localizations are more accurate when trained with smaller windows. Observations regarding No and Final Attention are similar to the past observations: the STDs are lower in the models with attention.

\subsubsection{Recall}

Results in Figure 8 show that localizations of models trained with 10k and 20k window sizes can discover breakpoints as good as models trained with $5 \mathrm{k}$ window size. Using too-large windows mostly results in degradation of Recall. Interestingly, localization of DeepSNPv1 No Attention trained with 40k can reveal nearly the same number of breakpoints compared with standard models trained with $5 \mathrm{k}$ as they achieve similar Recalls.

Looking at evaluations with larger window sizes of 10k and 20k provided in Figures 11 and 14, we observe similar patterns to the ones in $5 \mathrm{k}$ evaluations.

What can be concluded from evaluating models with $5 \mathrm{k}$ windows is that the localization output can reveal possible breakpoints. Their results in terms of breakpoint positions are satisfactory based on our evaluation results. The localization results of models trained with very large windows might introduce more false positives if evaluated with very high resolutions (small windows).

\section{CONCLUSION}

In this article, we proposed DeepSNP, a novel DNN trained in an end-to-end manner on SNPa data capable of classifying the presence or absence of one or multiple breakpoints within large genomic windows.

We demonstrated the capabilities of DeepSNP by comparing with state-of-the-art architectures as well as a known biological data analysis tool, Rawcopy. Our results showed that DeepSNP outperformed other deep models and could achieve performances that are in a reasonable range compared with specialized tools such as Rawcopy. We showed the ability of DeepSNP in producing annotation-like outputs for the presence/absence of breakpoints and further evaluated these localizations with various measures. We showed that the localizations can reveal the breakpoints up to a satisfactory level, although they introduce more false positives if evaluated with very high resolutions.

\section{FUTURE WORK}

In our future work, we will focus our efforts on improving the localization results by integrating strong labels in the training of DeepSNP. In addition, we will investigate the possibilities of the use of localization units for segmentation based on CNS transition. Finally, as DeepSNP was successful in processing SNPa, we will apply DeepSNP to other types of genomic data such as WGS.

\section{ACKNOWLEDGMENTS}

This work was carried out within the Austrian Research Promotion Agency (FFG) COIN "Networks" project VISIOMICS together with St. Anna Kinderkrebsforschung and additionally supported by the Austrian Ministry for Transport, Innovation and Technology, the Federal Ministry of Science, Research and Economy, and the Province of Upper Austria in the frame of the COMET center SCCH. The authors gratefully acknowledge the support of NVIDIA Corporation with the donation of a Titan X GPU used for this research. 


\section{AUTHOR DISCLOSURE STATEMENT}

The authors declare that no competing financial interests exist.

\section{REFERENCES}

Abadi, M., Barham, P., Chen, J., et al. 2016. Tensorflow: A system for large-scale machine learning. OSDI 16, 265283.

Ambros, I., Brunner, C., Abbasi, R., et al. 2014. Ultra-high density snparray in neuroblastoma molecular diagnostics. Front. Oncol. 4, 202.

Angermueller, C., Pärnamaa, T., Parts, L., et al. 2016. Deep learning for computational biology. Mol. Syst. Biol. 12, 878.

Benjamini, Y., and Speed, T.P. 2012. Summarizing and correcting the GC content bias in high-throughput sequencing. Nucleic Acids Res. 40, e72.

Ching, T.E.A. 2018. Opportunities and obstacles for deep learning in biology and medicine. J. R. Soc. Interface. 15, pii: 20170387.

Chollet, F., et al. 2015. Keras. https://keras.io

Chung, J., Gulcehre, C., Cho, K., et al. 2014. Empirical evaluation of gated recurrent neural networks on sequence modeling. arXiv arXiv:1412.3555.

Consortium, T.E.P. 2012. An integrated encyclopedia of DNA elements in the human genome. Nature 489, 57-74.

Eghbal-zadeh, H., Lehner, B., Dorfer, M., et al. 2016. Cp-jku submissions for dcase-2016: A hybrid approach using binaural i-vectors and deep convolutional neural networks. Presented at IEEE AASP Challenge on Detection and Classification of Acoustic Scenes and Events (DCASE). Budapest, Hungary.

Gupta, A., and Rush, A.M. 2017. Dilated convolutions for modeling long-distance genomic dependencies. arXiv arXiv: 1710.01278 .

Hershey, S., Chaudhuri, S., Ellis, D.P., et al. 2017. CNN architectures for large-scale audio classification. Presented at the IEEE International Conference on Acoustics, Speech and Signal Processing (ICASSP), 2017, New Orleans, LA, USA. pp. 131-135.

Hochreiter, S., and Schmidhuber, J. 1997. Long short-term memory. Neural Comput. 9, 1735-1780.

Huang, G., Liu, Z., van der Maaten, L., et al. 2016. Densely connected convolutional networks. arXiv arXiv: 1608.06993.

Kalchbrenner, N., Espeholt, L., Simonyan, K., et al. 2016. Neural machine translation in linear time. arXiv arXiv:1610.10099.

LaFramboise, T. 2009. Single nucleotide polymorphism arrays: A decade of biological, computational and technological advances. Nucleic Acids Res. 37, 4181-4193.

Marioni, J.C., Thorne, N.P., and Tavaré, S. 2006. BioHMM: A heterogeneous hidden Markov model for segmenting array CGH data. Bioinformatics 22, 1144-1146.

Mayrhofer, M., Viklund, B., and Isaksson, A. 2016. Rawcopy: Improved copy number analysis with Affymetrix arrays. Sci Rep. 6, 36158.

Olshen, A.B., Venkatraman, E., Lucito, R., et al. 2004. Circular binary segmentation for the analysis of array-based dna copy number data. Biostatistics 5, 557-572.

Pique-Regi, R., Monso-Varona, J., Ortega, A., et al. 2008. Sparse representation and Bayesian detection of genome copy number alterations from microarray data. Bioinformatics 24, 309-318.

Popitsch, N. 2018. Varan-gie: Curation of genomic interval sets. Bioinformatics. DOI: 10.1093/bioinFormatics/bty723.

Poplin, R., Newburger, D., Dijamco, J., et al. 2016. Creating a universal SNP and small indel variant caller with deep neural networks. Nature Biotechnology 36, 983-987.

Reddi, S.J., Kale, S., and Kumar, S. 2018. On the Convergence of Adam and Beyond. ICLR, Vancouver, Canada.

Simonyan, K., and Zisserman, A. 2014. Very deep convolutional networks for large-scale image recognition. arXiv arXiv:1409.1556.

Sun, Y.-Y., Zhang, Y., and Zhou, Z.-H. 2010. Multi-label learning with weak label. Presented at the Twenty-Fourth AAAI Conference on Artificial Intelligence. Atlanta, GA, USA.

Takahashi, N., and Mitsufuji, Y. 2017. Multi-scale multi-band densenets for audio source separation. Presented at the 2017 IEEE Workshop on Applications of Signal Processing to Audio and Acoustics (WASPAA), New York, NY, USA. pp. 21-25.

Van Den Oord, A., Dieleman, S., Zen, H., et al. 2016. Wavenet: A generative model for raw audio. arXiv arXiv:1609.03499.

Vaswani, A., Shazeer, N., Parmar, N., et al. 2017. Attention is all you need, pp. 6000-6010. In Advances in Neural Information Processing Systems. 
Venkatraman, E., and Olshen, A.B. 2007. A faster circular binary segmentation algorithm for the analysis of array CGH data. Bioinformatics 23, 657-663.

Wang, K., Li, M., Hadley, D., et al. 2007. Pennenv: An integrated hidden Markov model designed for high-resolution copy number variation detection in whole-genome SNP genotyping data. Genome Res. 17, 1665-1674.

Xu, Y., Kong, Q., Wang, W., et al. 2017. Large-scale weakly supervised audio classification using gated convolutional neural network. arXiv arXiv:1710.00343.

Yu, F., and Koltun, V. 2015. Multi-scale context aggregation by dilated convolutions. arXiv arXiv:1511.07122.

Zhang, Z.D., and Gerstein, M.B. 2010. Detection of copy number variation from array intensity and sequencing read depth using a stepwise Bayesian model. BMC Bioinformatics. 11, 539.

Zhao, M., Wang, Q., Wang, Q., et al. 2013. Computational tools for copy number variation (cnv) detection using nextgeneration sequencing data: Features and perspectives. BMC Bioinformatics. 14 Suppl 11, S1.

Address correspondence to:

Hamid Eghbal-zadeh, MSc

Institute of Computational Perception

Johannes Kepler University Linz

Altenberger Strasse 69

Linz 4040

Austria

E-mail: hamid.eghbal-zadeh@jku.at 